\title{
Identification of a Reliable Biomarker Profile for the Diagnosis of Gaucher Disease Type 1 Patients Using a Mass Spectrometry-Based Metabolomic Approach
}

\author{
Iskren Menkovic ${ }^{1}\left({ }^{1}\right.$, Michel Boutin $^{1}{ }^{(0)}$, Abdulfatah Alayoubi ${ }^{2,3}$, François E. Mercier ${ }^{2}$, \\ Georges-Étienne Rivard ${ }^{4}$ and Christiane Auray-Blais ${ }^{1, *}$ \\ 1 Division of Medical Genetics, Department of Pediatrics, Centre de Recherche-CHUS, Faculty of Medicine \\ and Health Sciences, Université de Sherbrooke, CIUSSS de l'Estrie-CHUS, 3001, 12th Avenue North, \\ Sherbrooke, QC J1H 5N4, Canada; iskren.menkovic@usherbrooke.ca (I.M.); \\ michel.boutin2@usherbrooke.ca (M.B.) \\ 2 Divisions of Experimental Medicine and Hematology, Department of Medicine, Faculty of Medicine, McGill \\ University, Lady Davis Institute for Medical Research, Jewish General Hospital, 3755, Côte Sainte-Catherine, \\ Montreal, QC H3T 1E2, Canada; abdulfatah.alayoubi@mail.mcgill.ca (A.A.); \\ francois.mercier@mcgill.ca (F.E.M.) \\ 3 Department of Biochemistry and Molecular Medicine, College of Medicine, Taibah University, University \\ Road, Madinah 42353, Saudi Arabia \\ 4 Division of Hemato-Oncology, Department of Pediatrics, Faculty of Medicine, Centre Hospitalier \\ Universitaire Sainte-Justine, 3175, Côte Sainte-Catherine, Montreal, QC H3T 1C5, Canada; \\ georges-etienne.rivard.hsj@ssss.gouv.qc.ca \\ * Correspondence: christiane.auray-blais@usherbrooke.ca
}

Received: 28 August 2020; Accepted: 20 October 2020; Published: 23 October 2020

\begin{abstract}
Gaucher disease (GD) is a rare autosomal recessive multisystemic lysosomal storage disorder presenting a marked phenotypic and genotypic variability. GD is caused by a deficiency in the glucocerebrosidase enzyme. The diagnosis of GD remains challenging because of the large clinical spectrum associated with the disease. Moreover, GD biomarkers are often not sensitive enough and can be subject to polymorphic variations. The main objective of this study was to perform a metabolomic study using an ultra-performance liquid chromatography system coupled to a time-of-flight mass spectrometer to identify novel GD biomarkers. Following the analysis of plasma samples from patients with GD, and age- and gender-matched control samples, supervised statistical analyses were used to find the best molecules to differentiate the two groups. Targeted biomarkers were structurally elucidated using accurate mass measurements and tandem mass spectrometry. This metabolomic study was successful in highlighting seven biomarkers associated with GD. Fragmentation tests revealed that these latter biomarkers were lyso- $\mathrm{Gb}_{1}$ (glucosylsphingosine) and four related analogs (with the following modifications on the sphingosine moiety: $-\mathrm{C}_{2} \mathrm{H}_{4},-\mathrm{H}_{2},-\mathrm{H}_{2}+\mathrm{O}$, and $+\mathrm{H}_{2} \mathrm{O}$ ), sphingosylphosphorylcholine, and N-palmitoyl-O-phosphocholineserine. Based on the plasma biomarker distribution, we suggest the evaluation of this GD biomarker profile, which might facilitate early diagnosis, monitoring, and follow-up of patients.
\end{abstract}

Keywords: Gaucher disease; metabolomics; mass spectrometry; glucosylsphingosine (lyso-Gb 1 ); lyso-Gb $\mathrm{G}_{1}$ analogs; N-palmitoyl-O-phosphocholineserine; sphingosylphosphorylcholine; biomarkers; plasma

\section{Introduction}

Lysosomal storage disorders (LSDs) are rare inherited metabolic conditions characterized by the inability of the lysosomes to process substrate(s), resulting in the accumulation of these compounds 
within the lysosome and ultimately leading to progressive and multisystemic clinical manifestations. Gaucher Disease type 1 (GD; OMIM 230800) is the most prevalent LSD with approximately 1 case in 40,000 births in the general population and 1 case in 800 births in the Ashkenazi Jewish population [1]. GD is the result of biallelic mutations in the GBA gene (1q22) leading to a deficiency of glucocerebrosidase, an enzyme involved in the catabolism of glucosylceramide $\left(\mathrm{Gb}_{1}\right)[1,2]$. This enzymatic defect leads to the accumulation of $\mathrm{Gb}_{1}$ in the lysosome of reticuloendothelial cells, ultimately resulting in the formation of Gaucher cells [3,4]. Cytopenia, hepatosplenomegaly, and bone lesions are usually observed in GD patients due to Gaucher cell's infiltration of the liver, spleen, and bone marrow $[4,5]$. From a clinical perspective, three subtypes of GD can be observed based on the absence, presence, and severity of neurological involvement. In Gaucher disease type 1, which is the most common form of the disease, representing $90-95 \%$ of all GD cases, patients may, in some cases, experience milder neurological impairments, whereas in GD types 2 and 3, patients experience more severe symptoms, such as abnormal eye movements and seizures, along with brain damage [6,7]. Type 2 Gaucher disease is usually observed in infancy, whereas type 3 GD is detected in childhood and tends to progress more slowly than GD type 2 [8]. However, it is worth noting that there are no clear distinctions between the type 2 and type 3 subtypes of GD but rather a phenotypic continuum ranging from horizontal ophthalmoplegia as the only neurological involvement in GD type 3 to hydrops fetalis in severe cases of GD type $2[5,9,10]$.

While enzyme replacement therapy (ERT) is the most frequently used treatment, substrate reduction therapy (SRT) may also be offered to patients [11]. While these treatments are effective to different extents, studies have demonstrated that early initiation of treatment, when required, will ultimately result in better outcomes for the patients [11]. However, due to the large clinical spectrum and heterogeneity of the disease, early detection and confirmation of the diagnosis of GD patients remain challenging, thus highlighting the need for reliable GD biomarkers. Biomarkers, such as C-C motif chemokine ligand (CCL18/PARC), angiotensin-converting enzyme (ACE), tartrate-resistant acid phosphatase $5 b$ (TRAP5b), and C-terminal telopeptide of type 1 collagen (CTx), may lead to the diagnosis of a GD case, but the quantitation of these compounds generally lack sensitivity and most of them are not specific to GD [12-16]. Chitotriosidase, an enzyme produced by activated macrophages, can be an interesting biomarker for the monitoring and follow-up of patients. However, it is not a reliable biomarker for the diagnosis of the disease, due to the high levels of polymorphism present in about $6 \%$ of the general population. This leads to major differences in baseline levels among GD patients, where some do not express chitotriosidase at all [17].

More recently, glucosylsphingosine (lyso-Gb $\mathrm{G}_{1}$ ), a substrate generated following the deacylation of glucosylceramide by lysosomal acid ceramidase [18], has been observed in the plasma of GD patients and has since gained interest in the medical and scientific community [19-21]. Unlike most of the previously mentioned biomarkers, lyso-Gb $\mathrm{G}_{1}$ is specific to GD and is potentially involved in the pathogenesis of the disease, more specifically regarding the immune system, as well as the skeletal and neurological systems [22]. Studies also tend to demonstrate that levels of lyso-Gb 1 can be useful biomarkers for the diagnostic, prognostic, monitoring, and follow up of GD patients [23]. Previous studies revealed that lyso-Gb $b_{1}$ in matrices such as dried blood spot or plasma can be reliable biomarkers for treatment efficiency and follow-ups of treated and untreated GD patients [24]. In fact, lyso-Gb $b_{1}$ has been evaluated for a median range of 22 years as part of a long-term follow up of a large cohort of GD patients [25]. While lyso-Gb b $_{1}$ appears to be a promising biomarker, we believe, based on research previously done by our group, that there might be other interesting biomarkers for GD patients that reflect the severity and progression of the disease in all GD patients. Indeed, previous metabolomic studies performed for Fabry disease, another lysosomal storage disorder, revealed that globotriaosylsphingosine $\left(l y s o-\mathrm{Gb}_{3}\right)$ is not a unique biomarker for Fabry disease patients.

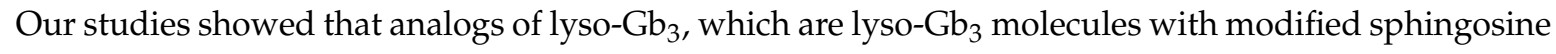
moieties, are present in both plasma and urine of Fabry patients and are relevant to the disease [26-29]. We therefore suggest that a similar GD pattern may occur, since both GD and Fabry disease are 
involved in the same metabolic pathway. Mirzain et al., hypothesized in 2015 that the chemical modifications observed on lyso- $\mathrm{Gb}_{3}$ to generate the analogs in Fabry disease (which were discovered by Auray-Blais et al.) may also be present on lyso-Gb $\mathrm{G}_{1}$ to generate the analogs in Gaucher disease [30]. Their hypothesis proved to be correct, and this led to the identification and quantification of some lyso-Gb $b_{1}$ analogs in GD plasma and urine specimens [30]. However, the compounds quantified by Mirzaian et al. were not characterized by mass spectrometry as being lyso-G $b_{1}$ analogs, since no fragmentation studies were performed. Moreover, the chromatographic method proposed by Mirzaian et al. was not able to separate lyso-Gb $b_{1}$ from galactosylsphingosine (psychosine), the latter being a biomarker for Krabbe disease [31]. Furthermore, compounds quantified by Mirzaian et al. were not subject to prior metabolomic studies, leading us to believe that other insightful molecules might be reliable biomarkers for GD.

The main objective of this study was to perform a semi-targeted plasma metabolomic study using ultra-performance liquid chromatography (UPLC) coupled to time-of-flight (TOF) mass spectrometry (MS) in order to identify a profile of sensitive and specific GD biomarkers that could be used for early detection, prognosis, monitoring, and follow up of affected patients. The secondary objective targeted the structural elucidation of biomarkers using accurate mass measurements and tandem mass spectrometry.

\section{Results and Discussion}

Before the development of the chromatographic separation method, we were aware of the existence of psychosine, a biomarker increased in Krabbe disease [32] which might interfere with the quantitation of lyso- $\mathrm{Gb}_{1}$ considering that it is a molecule with physical and chemical properties similar to lyso-Gb $b_{1}$ [33]. Therefore, in order to properly characterize the compound identified and better understand the physiopathology of Gaucher disease, a separation of psychosine and lyso-Gb is preferred. Since the two compounds are differentiated only by the conformation of one hydroxyl group, normal phase chromatography was chosen for their separation, as shown in Figure 1. Based on the retention times of these two compounds, it was possible to extrapolate the retention times of the lyso- $\mathrm{Gb}_{1}$ analogs based on their relative polarity, hence confirming that the analogs analyzed were from lyso-Gb $\mathrm{G}_{1}$ and not psychosine.

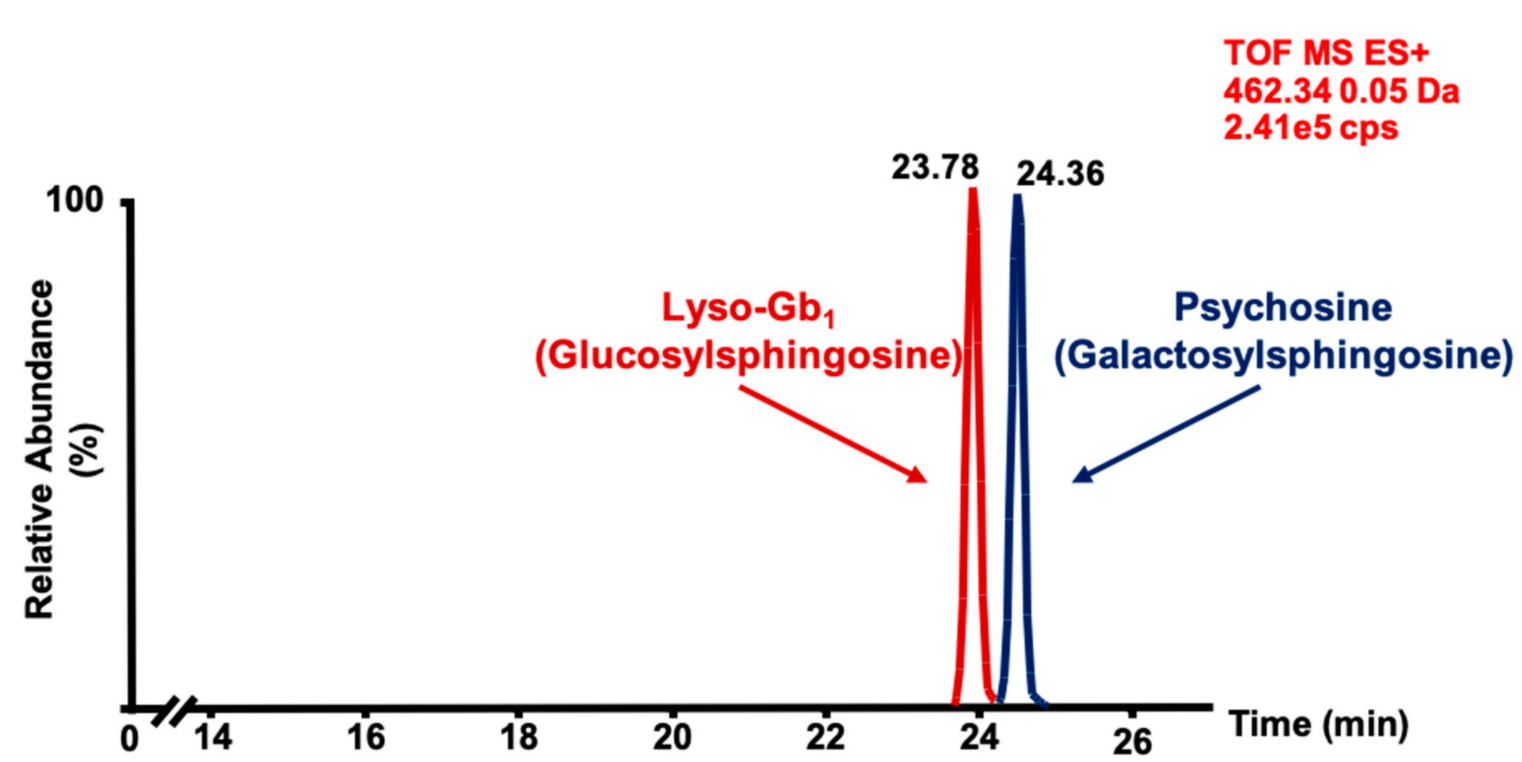

Figure 1. Chromatographic separation of glucosylsphingosine (lyso-Gb $1 ; 5 \mu$ on column, retention time: $23.78 \mathrm{~min}$ ) and psychosine ( $5 \mu \mathrm{g}$ on column, retention time: $24.36 \mathrm{~min}$ ) commercial standards analyzed by time-of-flight mass spectrometry (MS-TOF) with a mass window of $0.05 \mathrm{Da}$. Peaks associated with each compound were confirmed by individual analysis of each standard. ES+ = positive electrospray. 
Data mining steps with the use of MarkerLynx (version 4.1), following the analysis of 16 untreated GD patients and 16 age- and gender-matched healthy controls, yielded a total of 91,197 markers, which were aligned based on the retention time and accurate mass. Data were then imported in EZinfo for further multivariate statistical analyses. First, the system stability was evaluated using principal component analysis (PCA). Briefly, the PCA score plot allowed an evaluation of how similar or different samples were in terms of compositions according to their respective position on the score plot. Based on their chemical compositions, the more similar two samples were, the closer they appeared on the PCA score plot. Considering that quality controls (QCs) were replicate injections of a single sample, it was expected that QCs should be closely positioned on the PCA score plot. As shown in Figure 2, QCs $(n=8)$ (represented in red) were indeed very close, confirming that the system, as well as the samples were stable for the entire duration of the study. This also indicates that variations observed in metabolite concentrations in the samples analyzed were not system-related but rather due to biological reasons. Proximity in the location of QCs also indicates that data alignment parameters were adequate for the study. Moreover, considering that QCs were obtained by sampling and mixing equal volume $(20 \mu \mathrm{L})$ from each healthy control and GD patient sample, it was expected that QCs should be located in the center of the PCA score plot, as shown in Figure 2, also confirming the reliability of the analyses done during this study.

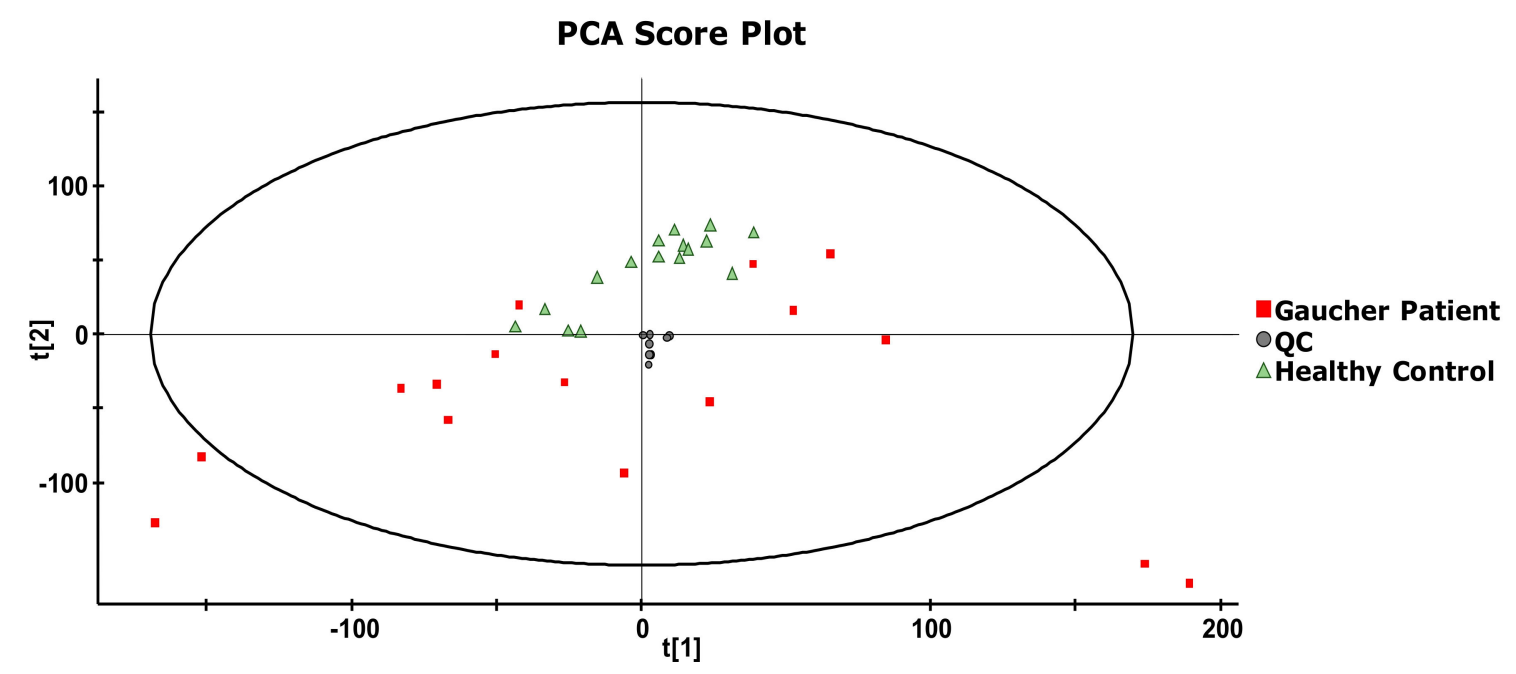

Figure 2. A principal component analysis (PCA) score plot derived from the metabolomic analysis of plasma samples from untreated Gaucher disease (GD) patients $(n=16)$, age- and gender-matched healthy controls $(n=16)$, and quality control $(\mathrm{QC})$ replicate injections $(n=8)$. The ellipse corresponds to the Hotelling T2 range with a significance level of $p=0.05$.

Once the system stability was confirmed, an orthogonal partial least-square discriminant analysis (OPLS-DA) was performed to identify compounds that would allow to separate the two groups under investigation. As shown in Figure 3, complete segregation of GD patients' samples and healthy control samples was achieved. It is important to note that based on the OPLS-DA chart, one patient was considered an outlier since he was plotted outside the Hotelling T2 range with a significance level of $p=0.05$. This patient was affected with a mild form of GD type 1 , which led to the system qualifying him as an outlier. However, he was not excluded from this study. 


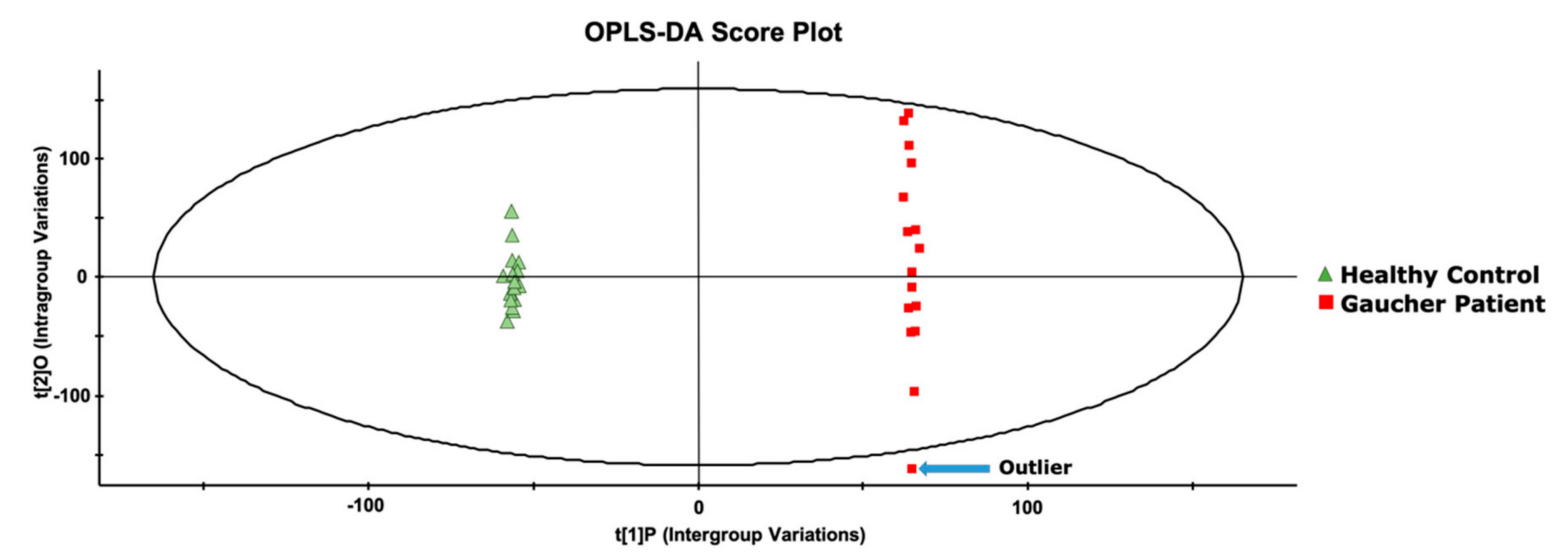

Figure 3. An orthogonal partial least-square discriminant analysis (OPLS-DA) score plot, resulting from the metabolomic analysis of plasma samples from untreated GD patients $(n=16)$ and age- and gender-matched healthy controls $(n=16)$. The ellipse corresponds to the Hotelling T2 range with a significance level of $p=0.05$.

The metabolites which contributed most to the segregation of the two sample groups on the OPLS-DA score plot were detected using an S-plot (Figure 4). The more a compound was abundant in GD samples compared to healthy control samples, further it was positioned in the upper right-side corner of the graph. Conversely, the more a compound was abundant in healthy control samples compared to GD samples, further it was positioned in the lower left-side corner of the graph. Metabolites showing the highest separation power were located at the extremities of the S-plot graph. While both sides of the graph were investigated to identify potential biomarkers for GD using trend plot charts (Figure A1), as well as a visual inspection of the chromatographic peak associated with the compound, only some metabolites located in the upper right-side corner of the graph ultimately resulted in potential GD biomarkers. Markers observed in less than 50\% of GD samples, or that were present only in GD samples collected from one hospital or detected at higher concentration in one or some control samples, compared to GD samples, were automatically discarded. Ultimately, 7 compounds with mass to charge ratios $(\mathrm{m} / \mathrm{z})$ of $434.3074,460.3227,462.3426,465.3428,476.3633,480.3553$, and 509.3335 were selected as potential GD biomarkers and further investigated using fragmentation tests to identify their structures (Figure 4).

To ensure that fragmentation tests would produce fragments of sufficient intensity to obtain their accurate mass, samples were further concentrated. While sample purification remained the same, samples were resuspended in $100 \mu \mathrm{L}$ instead of $250 \mu \mathrm{L}$ to provide a concentration factor of 2.5 for structural elucidation of all potential biomarkers, except analogs -24 and +14 , which were further concentrated five times to obtain a concentration factor of 12.5 . Based on its $m / z$, the compound $m / z 462.3426$ was suspected to be lyso- $\mathrm{Gb}_{1}$, which was later confirmed based on the fragmentation pattern. It was also observed that metabolites $\mathrm{m} / \mathrm{z} 434.3074,476.3633,480.3553$, and $460.3227 \mathrm{had}$ very similar fragmentation patterns to lyso- $\mathrm{Gb}_{1}$, suggesting that these four metabolites are analogs of lyso- $\mathrm{Gb}_{1}$. Based on the mass difference from lyso-Gb $\mathrm{b}_{1}$, it is believed that these four potential biomarkers are, in fact, lyso-Gb $\mathrm{Gb}_{1}$, with an extra double bond, which accounts for the loss of $2 \mathrm{H}(-2 \mathrm{Da})$ for metabolite $m / z 460.3227$, a loss of $\mathrm{C}_{2} \mathrm{H}_{4}(-28 \mathrm{Da})$ for metabolite $m / z 434.3074$, and a loss of $2 \mathrm{H}$ but a gain of $\mathrm{O}(+14 \mathrm{Da})$ for metabolite $\mathrm{m} / \mathrm{z} 476.3633$, and, finally, metabolite $\mathrm{m} / \mathrm{z} 480.3553$ possesses a hydrated sphingosine instead of a double-bond, which accounts for the gain of $18 \mathrm{Da}$. According to the fragmentation spectra obtained, all those proposed structural modifications were located on the sphingosine. In a recent study by Mirzaian et al. [30], the authors previously identified and quantified the following lyso-Gb $\mathrm{G}_{1}$ analogs $-28 \mathrm{Da},-2 \mathrm{Da}$, and $+14 \mathrm{Da}$, which were also highlighted in this study. However, the lyso-Gb $b_{1}$ analog at +18 Da has never been described in the literature.

The fragmentation pattern for each molecule and a general fragmentation mechanism for lyso-Gb is shown in Figure 5. 


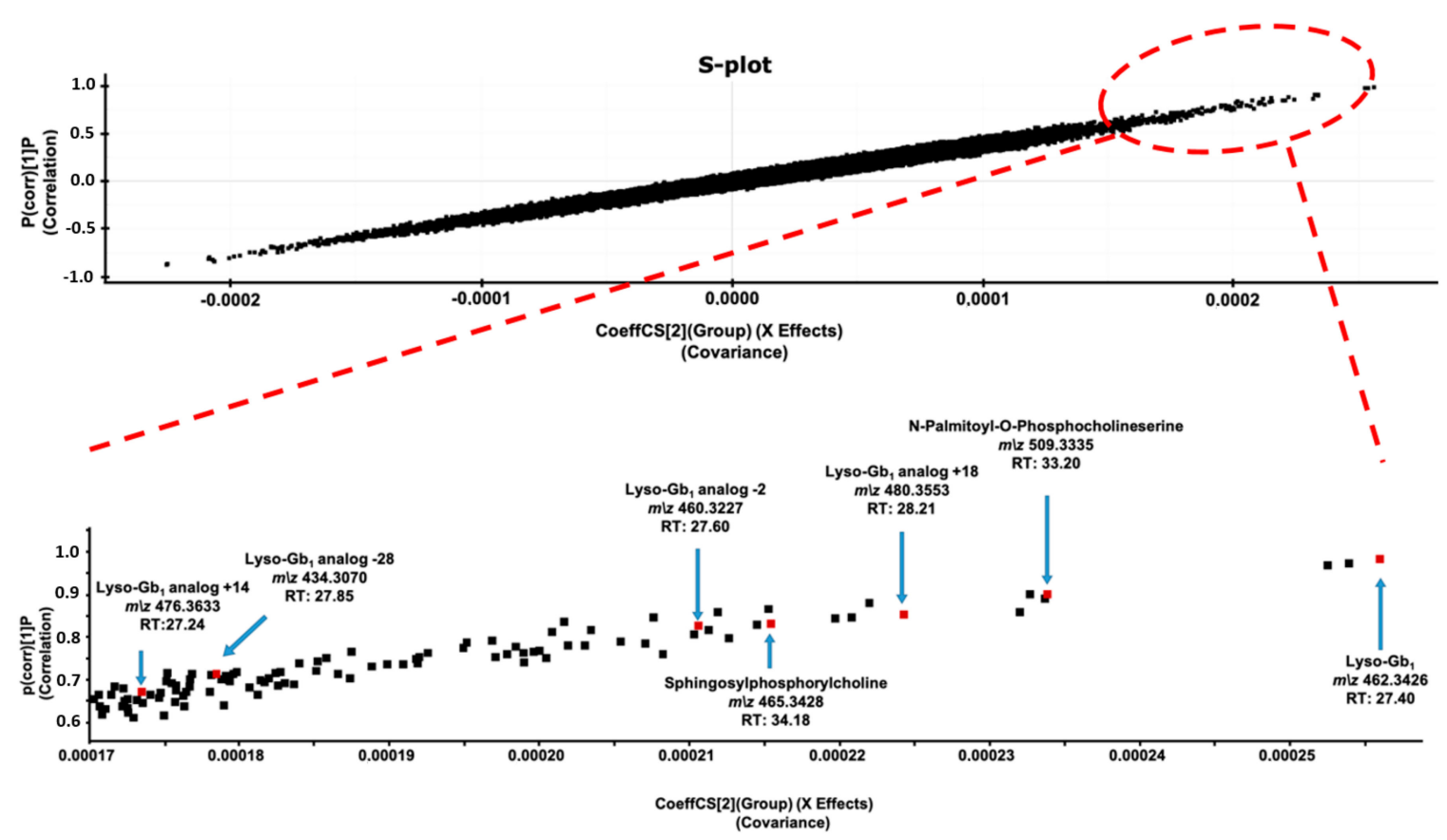

Figure 4. S-plot showing the correlation in function of the covariance for the metabolites used to segregate GD patients and age- and gender-matched healthy controls in the OPLS-DA score plot.

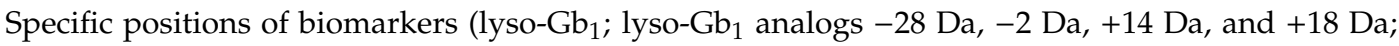
$\mathrm{N}$-palmitoyl-O-phosphocholineserine; and sphingosylphosphorylcholine) are zoomed in the lower part of the figure. Exact positions are indicated by a red square and an arrow. Accurate mass measured, as well as their respective retention times (RTs) in min, are indicated for each biomarker.

Following fragmentation tests, it was observed for biomarkers $\mathrm{m} / \mathrm{z} 465.3428$ and 509.3335 that a phosphocholine (PC) head group was present in both of these compounds based on the typical fragmentation pattern of PC heads, resulting in fragments with $\mathrm{m} / \mathrm{z}$ at 184.072, 125.000, and 104.110. However, excluding the PC head, the two aforementioned molecules had different fragmentation patterns, suggesting that they may not be as closely structurally related. Fragments from biomarker $\mathrm{m} / \mathrm{z} 509$ confirmed that it is a biomarker previously known as lyso-sphingomyelin-509 (lysoSM-509) (Figure 6).

Recently, Sidhu et al. proposed a structure for lysoSM-509 based on products obtained following precise chemical derivatization, aimed at confirming the presence of specific functional groups [34]. Ultimately, Sidhu et al. suggested that lysoSM-509 could be N-palmitoyl-O-phosphocholineserine. Fragments of the previously mentioned compound matched fragments observed in this study, confirming that the biomarker at $\mathrm{m} / z 509.3383$ is $\mathrm{N}$-palmitoyl-O-phosphocholineserine. However, Sidhu et al. also observed N-palmitoyl-O-phosphocholineserine in Niemann-Pick disease patients, suggesting that this latter biomarker is not a specific biomarker for Gaucher disease but rather an indicator of a sphingolipidosis [34,35]. 


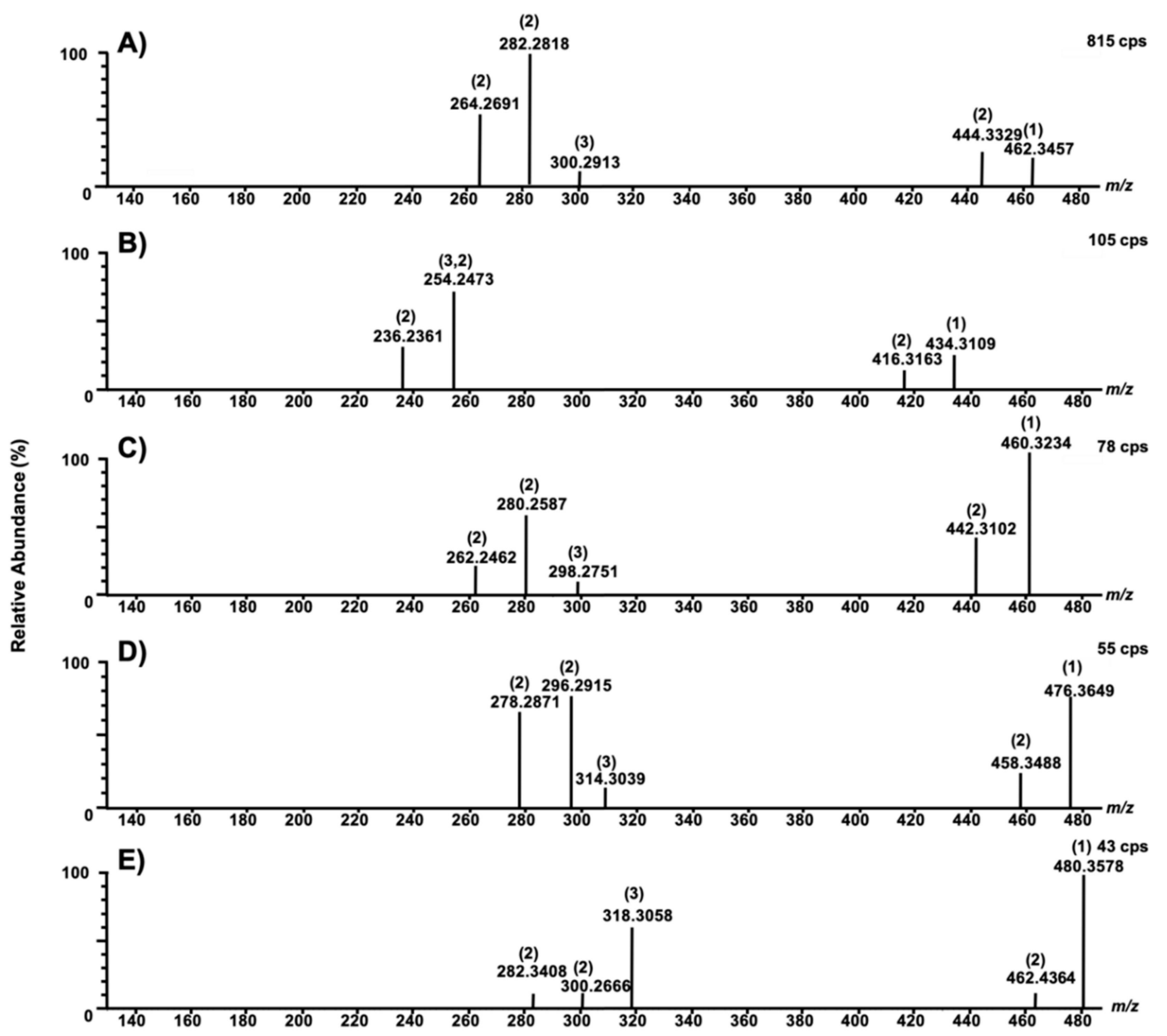

F)

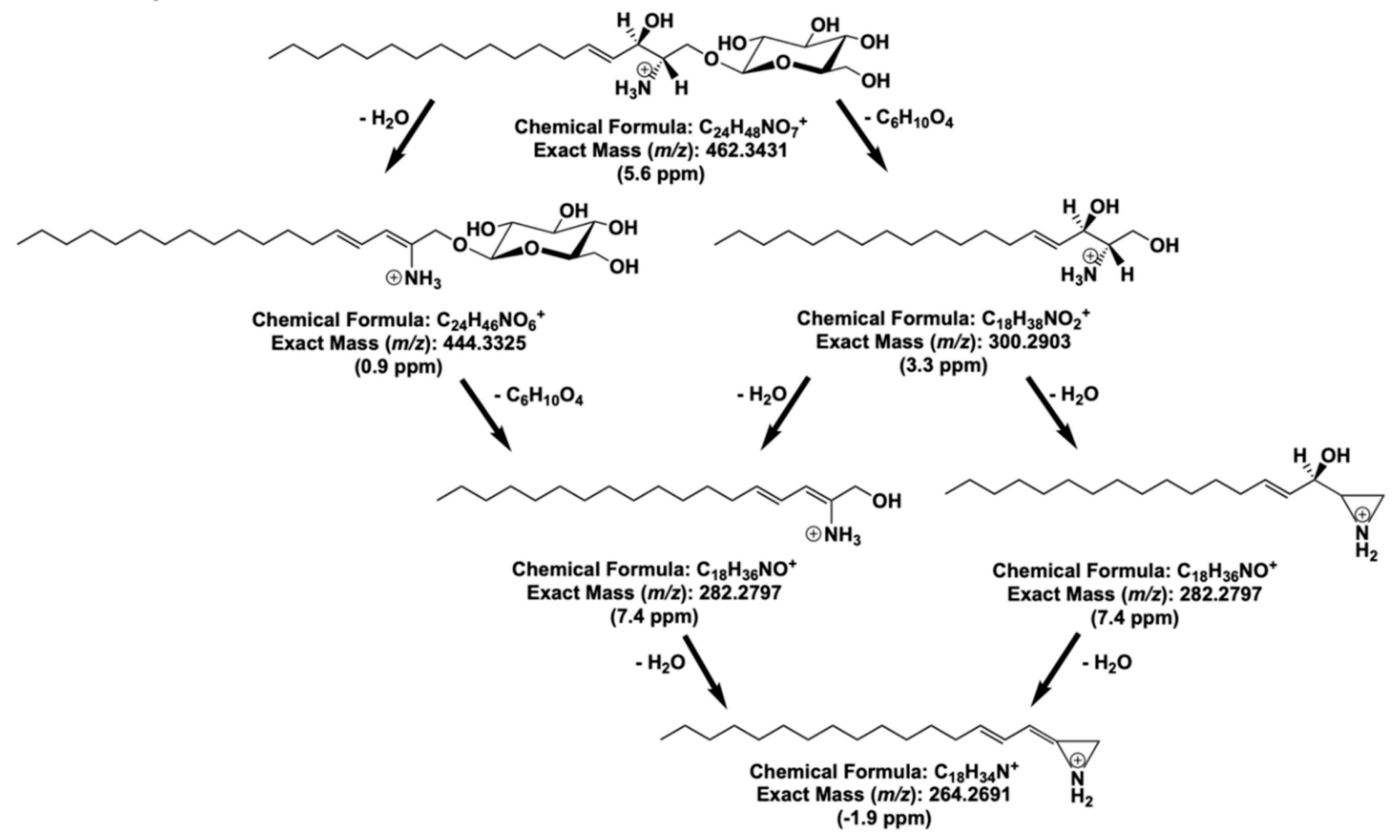

Figure 5. Fragmentation test results for compounds with $m / z 462.3426$ (A), 460.3227 (B), 480.3553 (C), 434.3074 (D), and 76.3633 (E), with a collision energy ramp of 10-30 V. In each spectrum, (1) corresponds to the precursor ion, (2) indicates a loss of a water molecule from the previous fragment, and (3) represents a loss of a molecule of glucose. A fragmentation mechanism for lyso-Gb $\mathrm{Gb}_{1}(\mathrm{~m} / \mathrm{z} 462.3227)$ is proposed in $(\mathbf{F})$. 
A)

$854 \mathrm{cps}$

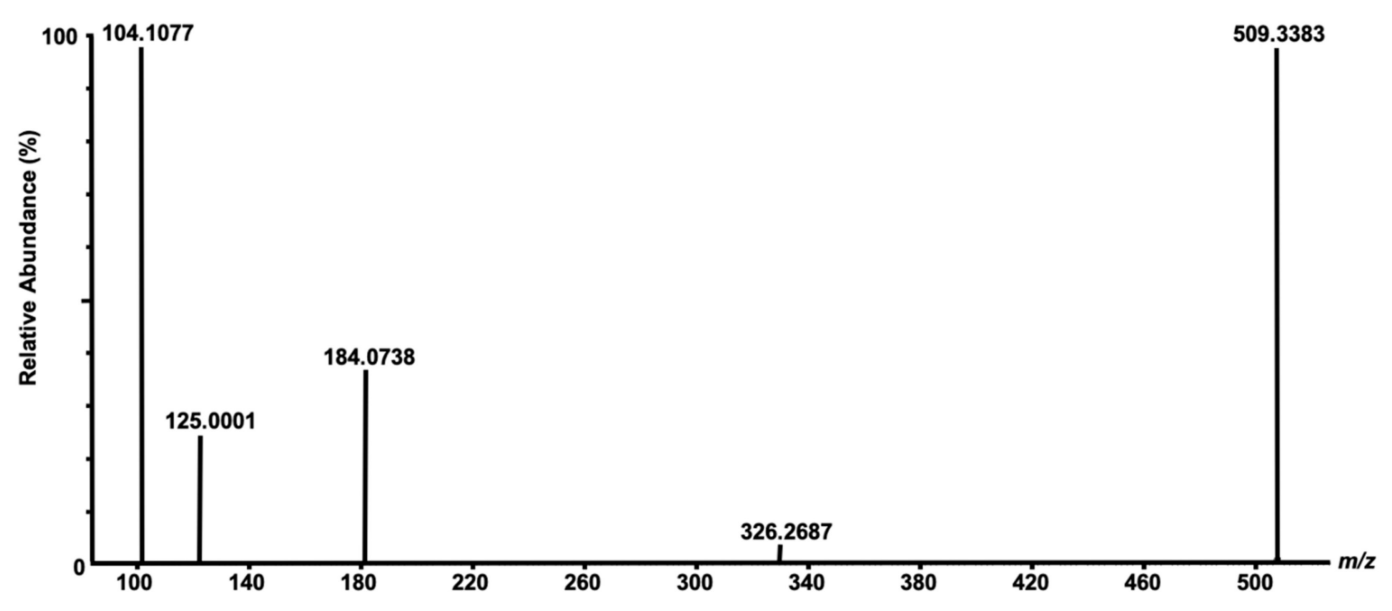

B)

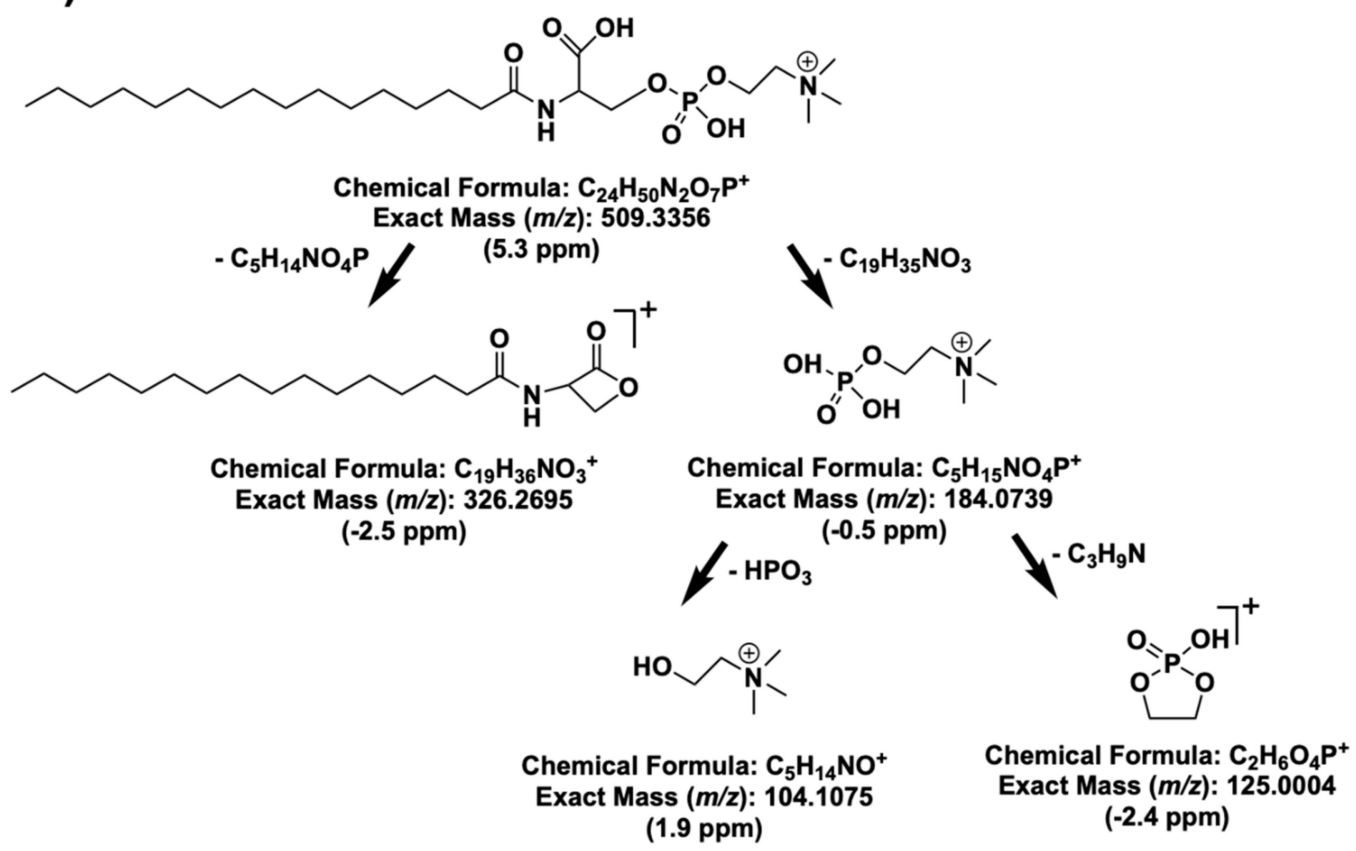

Figure 6. Fragmentation tests of compounds with $m / z 509$ with a collision energy ramp of 10-25 V (A) and a fragmentation mechanism previously proposed by Sidhu et al. [30], with theoretical masses and measured mass errors in part per million (ppm) (B).

Finally, the biomarker at $m / z 465$ was structurally elucidated based on the accurate mass measured and the fragmentation patterns (Figure 7). The proposed structure was ultimately confirmed by comparing fragments and their relative abundance, as well as the retention time of biomarker $\mathrm{m} / \mathrm{z} 465$ with fragments of a standard of sphingosylphosphorylcholine (SPC) (d18:1). 

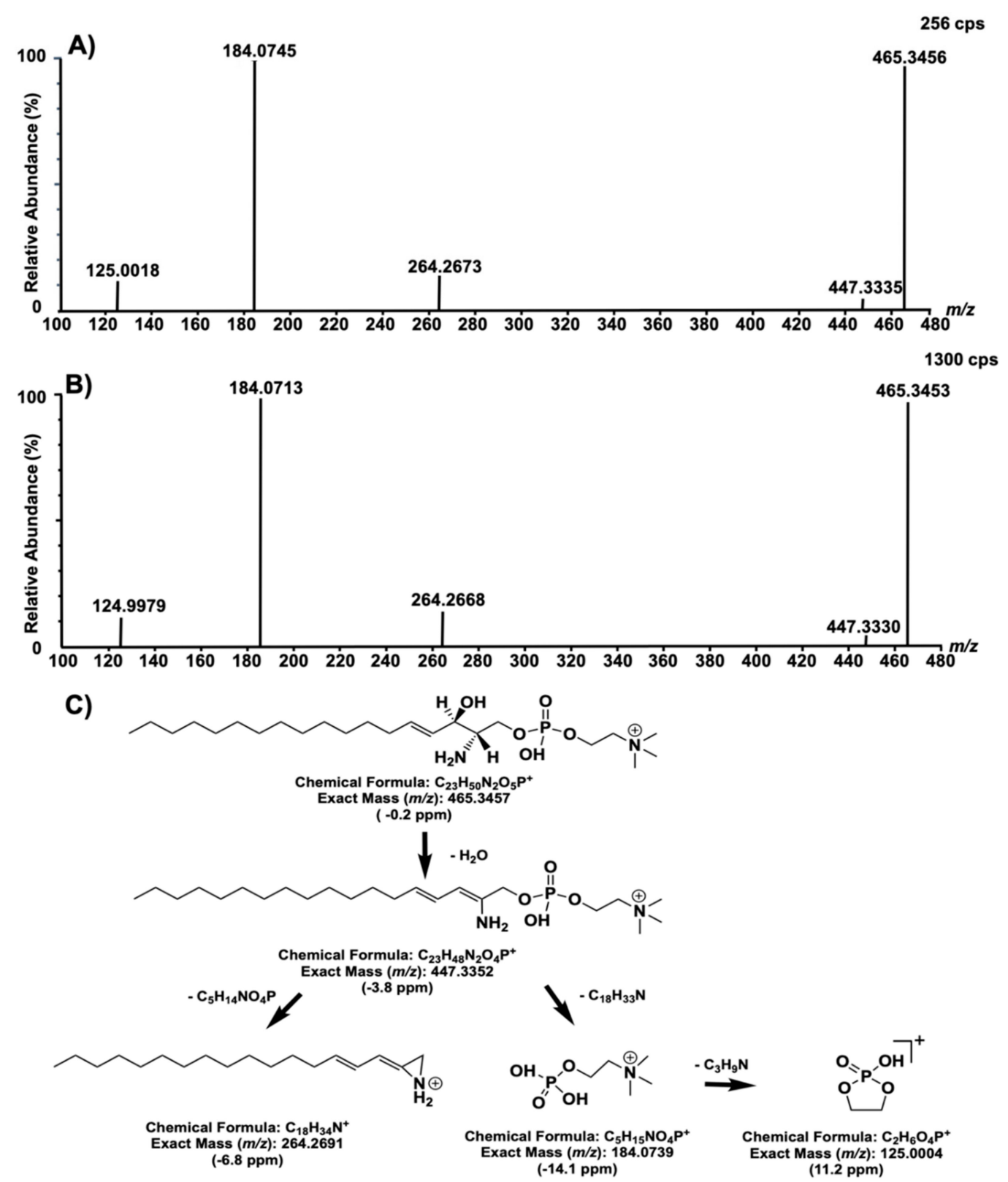

Figure 7. Fragmentation test results of compounds with $\mathrm{m} / \mathrm{z} 465$, with a collision energy ramp of 15-25 V (A). Fragmentation test results of a standard of sphingosylphosphorylcholine (d18:1) (10 $\mu \mathrm{g}$ on column) (B) and a proposed fragmentation mechanism with theoretical and experimental exact mass differences $(\mathbf{C})$.

Studies have demonstrated that SPC is a constituent of lipoproteins and a potentially important lipid mediator of cell type-specific functions in certain tissues. In fact, Thomas et al., have shown evidence that SPC in rats "is a powerful vasoconstrictor of intrapulmonary arteries (IPA) and the novel finding that SPC-induced vasoconstriction in IPA is dependent on activation of a $\mathrm{Ca}^{2+"}$ is important, since it can mediate certain cell mechanisms, such as contractility and cell proliferation [36]. Moreover, SPC may also be implicated in chronic vascular disease with involvement in processes such as proliferation and migration of vascular smooth muscle cells by acting as a pro-inflammatory mediator by activating p38 mitogen-activated protein kinases [36]. This is consistent with inflammation being involved in the pathogenesis of GD [37]. However, SPC (similar to N-palmitoyl-O-phosphocholineserine) is not specific to Gaucher disease. Indeed, significantly increased concentrations of SPC were observed in patients with acid sphingomyelinase deficiency such 
as Niemann-Pick type A and type B [38,39]. However, this metabolomic study showed the importance of SPC quantitation, even in GD patients considering the segregation power of the biomarker based on its position in the S-plot graph (Figure 4). More specifically, the increase of each potential biomarker identified during this metabolomic study is shown in Figure 8 for each GD patient. These potential biomarkers were not detected in healthy control samples.

A)

Bee-plot Distribution of Potential GD Biomarkers

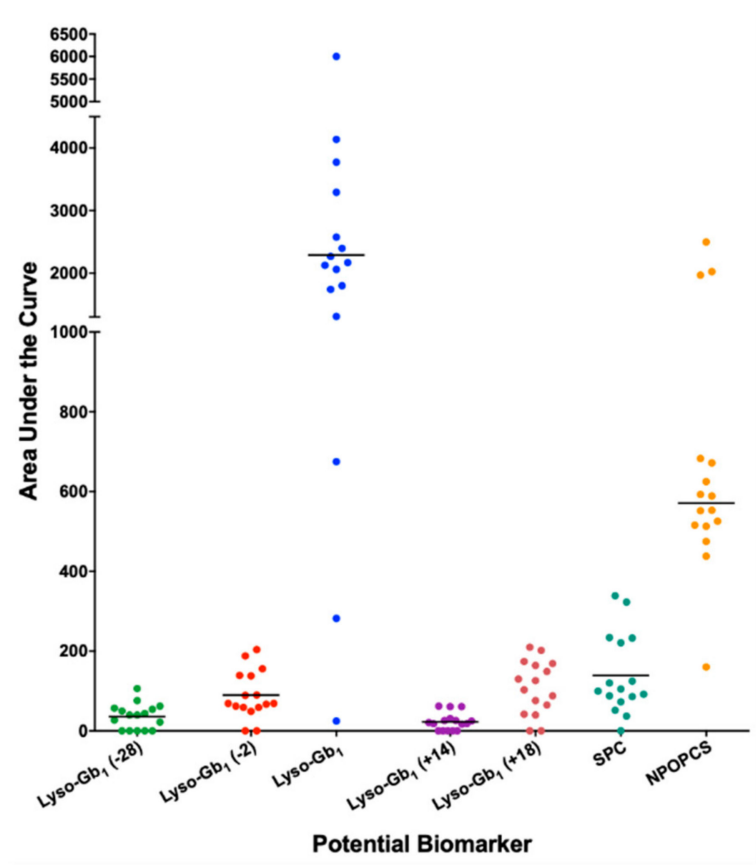

B) Cumulative Concentration of Potential GD Biomarkers

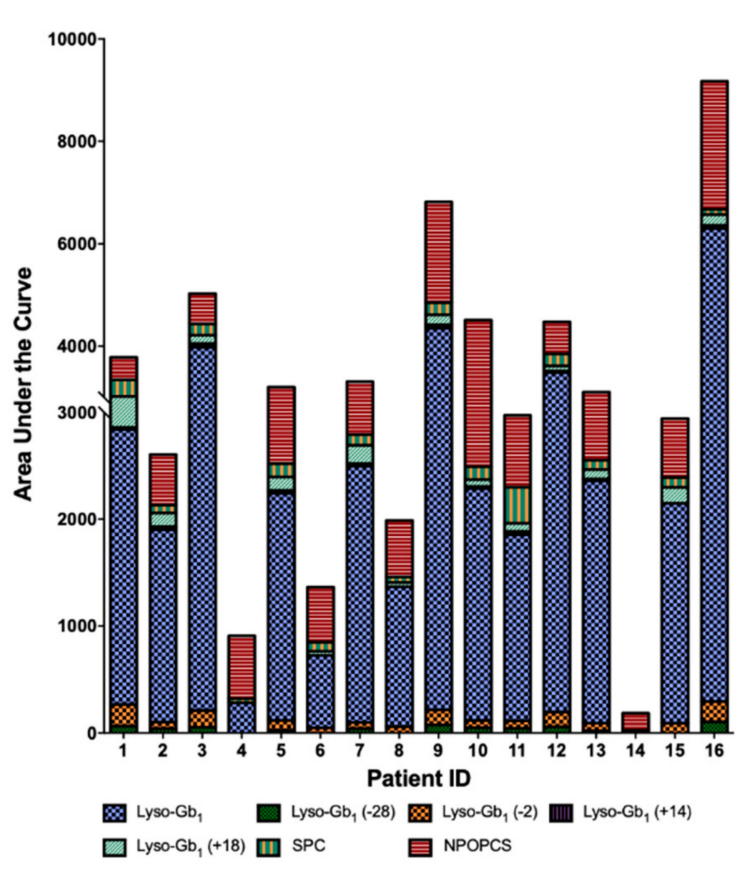

Figure 8. Bee-plot distribution (A) and cumulative concentrations (B) of potential GD biomarkers highlighted in the current metabolomic study. Sphingosylphosphorylcholine (SPC) and $\mathrm{N}$-palmitoyl-O-phosphocholineserine (NPOPCS). The line in (A) represents the median value for each potential biomarker. All potential GD biomarkers were not observed in healthy controls.

Our results show that N-palmitoyl-O-phosphocholineserine ( $\mathrm{G}$ in Figure A2) was the only marker to be elevated in all GD samples. An increase in the concentration of lyso-Gb ${ }_{1}$, a biomarker known for its diagnostic utility, as well as its monitoring performance in GD, was observed in all samples analyzed, except for one patient (ID 14), who was affected with a more attenuated form of the disease. [40,41]. While N-palmitoyl-O-phosphocholineserine seems to be a sensitive biomarker for GD, it is not specific to the disease, but is rather an indicator of a sphingolipidosis. N-palmitoyl-O-phosphocholineserine is also a biomarker for Niemann-Pick type C disease, while SPC is a biomarker for Niemann-Pick type A and type B diseases $[38,39,42]$. Therefore, a diagnostic of GD only based on that compound would not be possible.

Additionally, lyso-Gb $\mathrm{G}_{1}$ was significantly more abundant than all the other analogs in plasma.

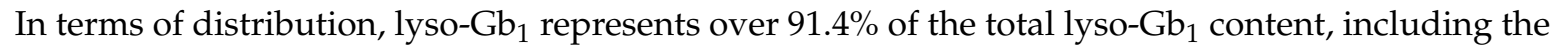
analogs. Meanwhile, the analogs at $-28 \mathrm{Da},-2 \mathrm{Da},+14 \mathrm{Da}$, and $+18 \mathrm{Da}$ represent approximately $1.6 \%$, $2.9 \%, 0.9 \%$, and $3.2 \%$ of the total lyso-Gb $-b_{1}$ analogs, respectively. The recovery of lyso-Gb $\mathrm{G}_{1}$ after solid phase extraction (SPE) was evaluated using a plasma sample spiked with $20 \mu \mathrm{g}$ of lyso-Gb $\mathrm{G}_{1}$ commercial standard, either pre $(n=3)$ or post-SPE $(n=3)$ purification. The recovery rate for lyso-Gb $\mathrm{b}_{1}$ was calculated to be over $92 \%$, thus suggesting that sample preparation was not a major source of lyso-Gb analog loss. While lyso-Gb $b_{1}$ seems to be a more abundant biomarker than its analogs in plasma for GD patients, we strongly believe that lyso-Gb $b_{1}$ and its related analogs should all be evaluated and quantified, since these analogs may correlate with certain specific clinical manifestations in GD, as found in Fabry disease [43]. There seems to be a correlation between the bone marrow burden (BMB) score 
observed by MRI and the levels of lyso-Gb 1 and its analogs. Moreover, for some patients, the levels of the analog at $-2 \mathrm{Da}$ were higher than those measured for the analog $+18 \mathrm{Da}$, while, for other patients, it was the opposite. Regarding SPC, an elevation of the biomarker was observed in all patients, except

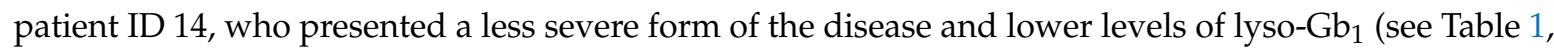
Figure A2). Unlike lyso-Gb ${ }_{1}$ and its analogs, as well as N-palmitoyl-O-phosphocholineserine, increased levels of SPC did not correlate with a more severe form of the disease. In fact, GD patient ID 16 had a particularly high BMD score, as shown in Table 1, compared to all other GD patients, yet his SPC levels were not significantly more elevated than other patients. Other compounds, such as glucosylceramide, a substrate normally catabolized by the enzyme glucocerebrosidase, which is deficient in GD patients, may potentially be an interesting biomarker for the disease. However, in order to investigate the relevancy of glucosylceramide and other similar compounds, a different sample preparation approach will be required. 
Table 1. Demographics, mutations, treatment, and signs and symptoms of GD patients. For bone marrow burden: lower limb (LL) and spine (S) score on 8. Düsseldorf Gaucher Score (DGS) score on 8. Non-applicable (N/A); not evaluated (N/E); (x) = sign or symptom observed in GD patient; (-) = sign or symptom not observed in GD patient; female (F); male (M). It should be noted that the samples sent from different hospitals had different evaluation tests and procedures for their respective patients. Moreover, certain tests, such as bone marrow burden, were not assessed for all patients.

\begin{tabular}{|c|c|c|c|c|c|c|c|c|c|c|c|c|c|c|c|c|}
\hline Patient ID & 1 & 2 & 3 & 4 & 5 & 6 & 7 & 8 & 9 & 10 & 11 & 12 & 13 & 14 & 15 & 16 \\
\hline Age & 62 & 49 & 43 & 62 & 22 & 47 & 49 & 70 & 63 & 71 & 62 & 43 & 70 & 53 & 24 & 44 \\
\hline Gender & $\mathrm{F}$ & $\mathrm{F}$ & $\mathrm{F}$ & $\mathrm{F}$ & $\mathrm{F}$ & $\mathrm{M}$ & $\mathrm{F}$ & $\mathrm{F}$ & $\mathrm{M}$ & $\mathrm{M}$ & $\mathrm{F}$ & $\mathrm{F}$ & $\mathrm{F}$ & $\mathrm{F}$ & $\mathrm{M}$ & $\mathrm{F}$ \\
\hline Age at diagnosis & 28 & 40 & 36 & 16 & $\mathrm{~N} / \mathrm{E}$ & 12 & 40 & 42 & 19 & 21 & 3 & 36 & $\mathrm{~N} / \mathrm{E}$ & 44 & 13 & 27 \\
\hline \multirow{2}{*}{ Mutations } & N370S & N370S & N370S & N370S & N370S & N370S & N370S & N370S & N370S I & N370S & L444P & N370S & N370S & N370S & N370S & L444P \\
\hline & N370S & N370S & L444P & N370S & W184 & L444P & N370S & L444P & R120W I & W378G & L444P & N370S & L444P & $\mathrm{R} 496 \mathrm{H}$ & L444P & $84 \mathrm{GG}$ \\
\hline Treatment & No & No & No & No & No & No & No & No & No & No & No & No & No & No & No & No \\
\hline \multicolumn{17}{|l|}{ Bone disease } \\
\hline Bone pain & - & Mild & Moderate & Mild & - & Moderate & - & - & - & Mild & Moderate & e Mild & Moderate & - & - & Mild \\
\hline Bone crisis & - & - & - & - & - & Moderate & - & - & Moderate & - & - & - & - & - & - & - \\
\hline Erlenmeyer flask deformity & $\mathrm{x}$ & - & - & - & - & & - & - & - & & - & $\mathrm{x}$ & $\mathrm{x}$ & - & - & $\mathrm{x}$ \\
\hline Bone marrow infiltration & $\mathrm{x}$ & $\mathrm{x}$ & $\mathrm{x}$ & $\mathrm{x}$ & - & $\mathrm{x}$ & - & - & - & $\mathrm{x}$ & - & $\mathrm{x}$ & $\mathrm{x}$ & - & $\mathrm{x}$ & $\mathrm{x}$ \\
\hline Osteopenia & - & - & - & - & - & $\mathrm{x}$ & - & - & $\mathrm{x}$ & $\mathrm{x}$ & $\mathrm{x}$ & - & $\mathrm{x}$ & $\mathrm{x}$ & - & - \\
\hline Infarction & - & - & - & - & - & $\mathrm{x}$ & - & - & $\mathrm{x}$ & - & - & - & $\mathrm{x}$ & - & - & $\mathrm{x}$ \\
\hline Avascular necrosis & - & - & - & - & - & $\mathrm{x}$ & - & - & - & - & - & - & - & - & - & - \\
\hline New fractures & - & - & - & - & - & - & - & - & - & - & - & - & - & - & - & - \\
\hline Lytic lesions & $\mathrm{x}$ & - & - & - & - & - & - & - & $\mathrm{x}$ & - & - & - & - & - & - & - \\
\hline Decreased bone mineral density & - & - & - & - & - & $\mathrm{x}$ & - & - & - & $x$ & - & - & - & $\mathrm{x}$ & - & - \\
\hline Bone marrow burden & DGS 5 & LL 5 S 6-7 & $\mathrm{N} / \mathrm{E}$ & LL 4 S 3 & $\mathrm{~N} / \mathrm{E}$ & LL 4 S 3 & $\mathrm{~N} / \mathrm{E}$ & LL 7 S 5 & DGS 7 & $\mathrm{~N} / \mathrm{E}$ & $\mathrm{N} / \mathrm{E}$ & LL 4 S 5-6 & DGS 7 & LL 2 S 3 & $\mathrm{~N} / \mathrm{E}$ & LL 7 S 7 \\
\hline \multicolumn{17}{|l|}{$\begin{array}{l}\text { Hematological and visceral } \\
\text { manifestations }\end{array}$} \\
\hline Anemia & - & - & $\mathrm{x}$ & - & - & - & - & - & $x$ & - & $x$ & $x$ & - & - & - & - \\
\hline Hemoglobin $(\mathrm{g} / \mathrm{L})$ & 125 & 125 & 118 & 140 & 127 & 149 & 122 & 132 & 103 & 140 & 110 & 113 & 123 & 117 & 124 & 127 \\
\hline $\begin{array}{l}\text { Thrombocytopenia and } \\
\text { coagulopathy }\end{array}$ & - & $\mathrm{x}$ & $\mathrm{x}$ & $\mathrm{x}$ & - & - & $\mathrm{x}$ & - & $\mathrm{x}$ & - & - & $\mathrm{x}$ & $\mathrm{x}$ & - & $\mathrm{x}$ & $\mathrm{x}$ \\
\hline Platelet counts (/L) & 156 & 87 & 65 & 95 & 123 & 197 & 117 & 144 & 63 & 182 & 185 & 82 & 130 & 254 & 103 & 71 \\
\hline Splenomegaly & Mild & Mild & Mild & - & - & - & $\mathrm{x}$ & N/A & Moderate & N/A & N/A & - & N/A & - & - & N/A \\
\hline Hepatomegaly & - & Mild & Mild & Mild & - & Mild & - & Mild & Moderate & $\mathrm{N} / \mathrm{E}$ & Mild & Moderate & Mild & Moderate & Severe & - \\
\hline Splenectomized & - & - & - & - & - & - & - & $x$ & - & $\mathrm{x}$ & $x$ & - & $x$ & - & - & $x$ \\
\hline Gammopathies and malignancy & - & - & - & - & - & - & - & - & - & $x$ & - & - & - & - & - & - \\
\hline Pulmonary disease & & & & & & - & - & & & & & & & & & \\
\hline Elevated arterial pressure & - & - & - & - & - & - & - & - & - & $\mathrm{x}$ & $\mathrm{x}$ & - & - & - & - & - \\
\hline Severe pulmonary hypertension & - & - & - & - & - & - & - & - & - & - & - & - & - & - & - & - \\
\hline Hepatopulmonary syndrome & - & - & - & - & - & - & - & - & - & - & - & - & - & - & - & - \\
\hline Respiratory failure & - & - & - & - & - & - & - & - & - & - & - & - & - & - & - & - \\
\hline Other & - & - & - & - & - & - & - & - & - & - & - & - & - & - & - & - \\
\hline
\end{tabular}




\section{Materials and Methods}

\subsection{Ethics Approval}

This project was approved by the Research Ethics Board (REB) of the Faculty of Medicine and Health Sciences of the Centre Hospitalier Universitaire de Sherbrooke (CHUS: Project ID MP-31-2017-1414, 28th September 2016), as well as the REB of Montreal's Jewish General Hospital (Project ID MEO-31-2020-1937, 22nd November 2019) and Centre Hospitalier Universitaire Sainte-Justine as a multicentric evaluation (Project ID MEO-31-2021-2782, 28th September 2016). Informed consent from all GD patients and healthy controls were obtained.

\subsection{Patients and Controls}

Blood samples were collected from 16 untreated patients previously diagnosed with GD (age range: 23-68 years old; 8 males, 8 females) and 16 age- and gender-matched controls (age range: 26-66 years old; 9 males, 7 females). Adult men and women with a confirmed diagnosis of GD type 1 were included in this study. Treated GD type 1 patients, as well all GD type 2 and type 3 patients, were excluded from this study. Controls recruited did not have any LSDs or other comorbidities. The diagnosis was confirmed for each GD patient by demonstrating marked enzyme deficiency of $\beta$-glucosidase in peripheral blood leukocytes and sequencing of the GBA gene. Only untreated GD patient samples were considered for this metabolomic study to ensure that a significant increase of potential GD biomarkers would be observed.

Clinical Features and Biochemical Parameters

GD patients' demographics, mutations, treatment, and signs and symptoms are shown in Table 1. Some patients had comorbidities, such as diabetes (patients 4 and 11), ovarian adenocarcinoma (patients 8 and 13), and hypercholesterolemia (patients 4 and 10). Patient 6 had unconjugated hyperbilirubinemia, while patient 9 was suffering from minor beta thalassemia and multiple cystic lesions in the liver and spleen.

\subsection{Whole Blood and Plasma Specimen Collection and Processing}

Using a $21 \mathrm{G}$ butterfly needle, whole blood samples were collected into BD Vacutainer tubes with sodium citrate as an anticoagulant. Plasma was immediately extracted by centrifugation at $2000 \times g$ for $10 \mathrm{~min}$ and transferred into $15 \mathrm{~mL}$ Falcon tubes. Plasma samples were stored at $-80{ }^{\circ} \mathrm{C}$ until the metabolomic study was performed.

\subsection{Reagents}

Glucosysphingosine (lyso-Gb $\mathrm{G}_{1} ;>98 \%$ ) and galactosylsphingosine (Psychosine; >98\%) were purchased from Matreya (Pleasant Gap, PA, USA). LC-MS grade acetonitrile (ACN) and HPLC grade methanol $(\mathrm{MeOH})$ were purchased from EMD Chemicals Inc. (Darmstadt, Germany), while Optima LC-MS grade water, American Clinical Society (ACS) grade ammonium formate (Amm. Form.), o-phosphoric acid $\left(\mathrm{H}_{3} \mathrm{PO}_{4} ; 85 \%\right)$, and ammonium hydroxide $\left(\mathrm{NH}_{4} \mathrm{OH} ; 28 \%-30 \%\right.$ purity) were obtained from Fisher Scientific (Fair Lawn, NJ, USA). ReagentPlus grade dimethyl sulfoxide (DMSO; $\geq 99.5 \%$ ) and terfenadine were obtained from Sigma-Aldrich (St. Louis, MO, USA), and, finally, formic acid (FA; >99\%) was purchased from Acros Organics (Morris Plain, NJ, USA).

\subsection{Sample Preparation}

Untreated GD patients or healthy control plasma samples were vortexed for $10 \mathrm{~s}$, and $200 \mu \mathrm{L}$ of plasma was then transferred into $2 \mathrm{~mL}$ Eppendorf plastic tubes. A total of $500 \mu \mathrm{L}$ of $\mathrm{H}_{3} \mathrm{PO}_{4}(2 \%$ in water) and $500 \mu \mathrm{L}$ of $\mathrm{MeOH}$ were added to the plasma sample, followed by $10 \mathrm{~s}$ of mixing (vortex). Sample mixtures were transferred to mixed-mode cation-exchange cartridges (Oasis MCX, 30 mg, 
$60 \mu \mathrm{m}$, Waters Corp., Milford, MA, USA), previously conditioned with $1000 \mu \mathrm{L}$ of $\mathrm{MeOH}$ and $1000 \mu \mathrm{L}$ of $2 \% \mathrm{H}_{3} \mathrm{PO}_{4}$ using a glass pipette. Cartridges were washed with $1000 \mu \mathrm{L}$ of $\%$ FA in $\mathrm{H}_{2} \mathrm{O}$ solution, followed by $1000 \mu \mathrm{L}$ of $0.2 \% \mathrm{FA}$ in $\mathrm{MeOH}$ solution. Analytes were then eluted into glass tubes using $600 \mu \mathrm{L}$ of $2 \%$ ammonium hydroxide in $\mathrm{MeOH}$ solution. Eluates were evaporated to dryness under a stream of nitrogen. Finally, samples were resuspended in $250 \mu \mathrm{L}$ of the resuspension solution $(50 \mu \mathrm{L}$ DMSO mixed with $200 \mu \mathrm{L}$ of 94.5:2.5:2.5:0.5 ACN:MeOH: $\mathrm{H}_{2} \mathrm{O}: \mathrm{FA}+5 \mathrm{mM}$ Amm. Form) and transferred into glass inserts fitted into $2 \mathrm{~mL}$ glass vials. A total of $10 \mu \mathrm{L}$ of the sample was injected into the UPLC-TOF/MS system for analysis. A quality control (QC) sample was prepared by mixing together $20 \mu \mathrm{L}$ of each sample from GD patients $(n=16)$ and healthy controls $(n=16)$ analyzed in the study.

\subsection{Instrumentation and Parameters}

The metabolomic study was performed using the Synapt G1 QTOF (Waters Corp., Milford, MA, USA) mass spectrometer coupled to an Acquity UPLC system (Waters Corp). Chromatographic separation of plasma components was achieved in a 45-min method using a HPLC Halo HILIC column $(4.6 \times 150 \mathrm{~mm}, 2.7 \mu \mathrm{m}$ particle diameter) from Advanced Materials Technology (Wilmington, DE, USA), with a constant flow rate set at $0.5 \mathrm{~mL} / \mathrm{min}$ and an operating temperature of $30^{\circ} \mathrm{C}$. Chromatographic mobile phase A was 94.5:2.5:2.5:0.5 ACN:MeOH: $\mathrm{H}_{2} \mathrm{O}: \mathrm{FA}+5 \mathrm{mM} \mathrm{Amm}$. Form., while chromatographic mobile phase B was 10:89.5:0.5 ACN: $\mathrm{H}_{2} \mathrm{O}: \mathrm{FA}+5 \mathrm{mM}$ Amm. Form. The "weak" and "strong" needle wash solutions were Phase A and 100\% ACN, respectively. The gradient and in-depth parameters used to separate sample components are presented in Table 2.

Table 2. Ultra-performance liquid chromatography (UPLC) parameters for the separation of the plasma samples analyzed during the metabolomic study.

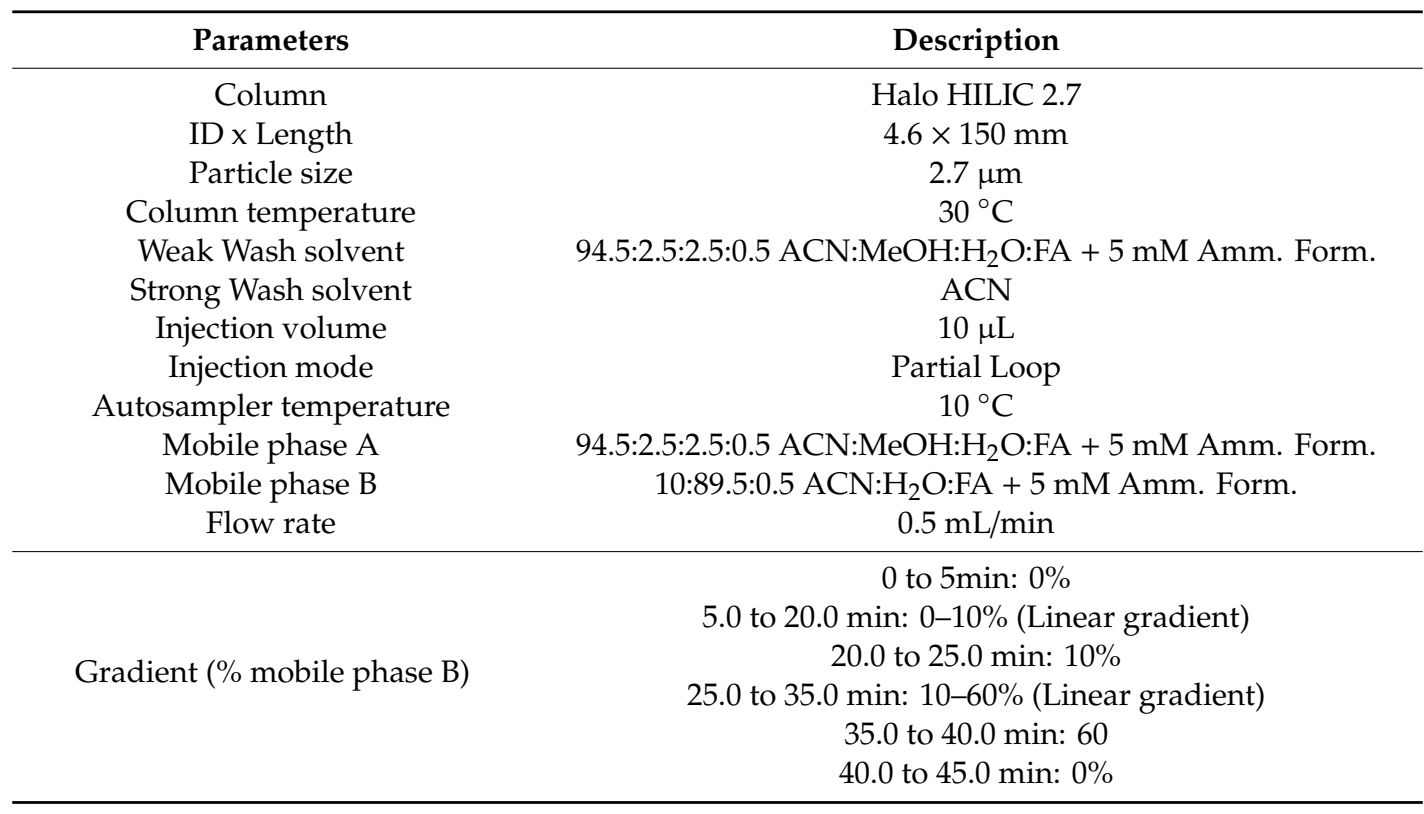

The parameters for data acquisition, lock mass calibration, and MS-TOF are presented in Table 3. In order to maintain a high level of mass accuracy throughout the entire duration of the metabolomic run analysis, a standard solution of terfenadine (500 nM, ACN 5\%, FA $0.2 \%)$, used for real-time calibration, was continuously infused throughout the analysis, using a lock mass probe connected to an isocratic pump model 515 (Waters Corp.). The flow rate of the isocratic pump was set at $1 \mathrm{~mL} / \mathrm{min}$. However, since a $\mathrm{T}$ splitter was used in the system, linking the pump to the lock mass probe, the actual flow rate of calibrant used was approximately $12 \mu \mathrm{L} / \mathrm{min}$. 
Table 3. Mass spectrometry (MS) parameters for the quantitation of compounds present in the plasma samples analyzed during the metabolomic study.

\begin{tabular}{|c|c|}
\hline Parameters & Description \\
\hline Scan Mode & MS-TOF \\
\hline Ionization Mode & Electrospray Ionization \\
\hline Polarity & Positive \\
\hline Analyzer Mode & $\mathrm{V}$ \\
\hline Dynamic Range & Extended \\
\hline Capillary Voltage & $1.4 \mathrm{kV}$ \\
\hline Sampling Cone Voltage & $10 \mathrm{~V}$ \\
\hline Extraction Cone Voltage & $5.0 \mathrm{~V}$ \\
\hline Source Temperature & $150^{\circ} \mathrm{C}$ \\
\hline Desolvation Temperature & $450{ }^{\circ} \mathrm{C}$ \\
\hline Cone Gas Flow & $30 \mathrm{~L} / \mathrm{h}$ \\
\hline Desolvation Gas Flow & $700 \mathrm{~L} / \mathrm{h}$ \\
\hline Trap Collision Energy & $4.0 \mathrm{~V}$ \\
\hline Transfer Collision Energy & $2.0 \mathrm{~V}$ \\
\hline Data Format & Centroid \\
\hline Mass Range & $50-1000 \mathrm{Da}$ \\
\hline Scan Time & $0.1 \mathrm{~s}$ \\
\hline \multicolumn{2}{|c|}{ Lock mass } \\
\hline Compound & Terfenadine (500 nM) \\
\hline Exact Mass & $472.3215 \mathrm{Da}$ \\
\hline Solvent & 94.5:5:0.5 $\mathrm{H}_{2} \mathrm{O}: \mathrm{ACN}: \mathrm{FA}$ \\
\hline Scan Time & $0.5 \mathrm{~s}$ \\
\hline Interval & $5.0 \mathrm{~s}$ \\
\hline Sampling Cone Voltage & $5 \mathrm{~V}$ \\
\hline Trap Collision Energy & 10 \\
\hline Mass Window & $\pm 0.2 \mathrm{Da}$ \\
\hline Scan Average & 3 \\
\hline
\end{tabular}

Following the UPLC-TOF/MS analysis, the peaks detected were processed using the software MarkerLynx XS (Waters Corp., Version 4.1) in order to align markers detected in samples based on their UPLC retention times and mass to charge ratios $(\mathrm{m} / \mathrm{z})$ and to evaluate their peak areas. Markers with a mass below $50 \mathrm{Da}$ and above $1000 \mathrm{Da}$ were also excluded in this step. Based on data obtained from the QC samples analyzed periodically throughout the metabolomic run in alternance with GD patient samples and healthy control samples, the mass window and the retention time window were set at $0.05 \mathrm{Da}$ and $0.20 \mathrm{~min}$. The intensity threshold was set at 5 counts. Data from over 91,000 different peaks detected in the samples analyzed were then transferred to EZinfo, a software of Umetrics (Umeå, Sweden) integrated to the Extended Statistics Tools Box of MassLynx v4.1 (Waters Corp.), for multivariate statistical analysis in order to identify compounds expressed differently in the GD patients group compared to the healthy control group. The Unit Variance (UV) and Pareto scaling algorithms, with and without logarithmic transformation, were tested.

Ultimately, UV scaling with logarithmic transformation was selected, since all the most promising biomarkers highlighted with the other scaling methods were also detected with this algorithm. UV scaling is expressed by the equation $\mathrm{UV}=\frac{x-\bar{x}}{S D}$, where $x$ is the area under the curve for a marker in a specific sample, whereas $\bar{x}$ and SD are the average and the standard deviation of the areas under the curve for the same marker measured in all samples, respectively. UV scaling reduces the emphasis of more abundant compounds while increasing the contribution of less abundant compounds, making all metabolites similarly important regardless of their respective abundance [44]. Logarithmic transformation, on the other hand, is used in order the correct the heteroscedasticity observed in the analyses [44]. Once an optimal scaling method was selected, the system stability throughout the study was inspected by evaluating the distribution of QC sample replicate injections $(n=8)$ performed 
periodically during the metabolomic run in a principal component analysis (PCA) chart. Following PCA analysis, an orthogonal partial least-square discriminant analysis (OPLS-DA) was used to target compounds with differential expression in the two groups under investigation. Finally, an S-plot was used to select metabolites contributing the most to the discrimination between the GD patients' samples and the healthy control samples during OPLS-DA. Based on the trend plot chart distributions, metabolites that were expressed differently in the two groups were selected and further investigated.

Fragmentation tests using UPLC-quadrupole time-of-flight (QTOF) mode were performed to structurally elucidate the compounds of interest. These compounds were isolated in the quadrupole and fragmented in the collision cell, and the produced fragments were analyzed using the TOF analyzer to obtain their accurate masses. Main source ionization parameters and UPLC parameters remained unchanged for this procedure, while lock mass sampling cone voltage was increase to 12 to obtain a good signal for terfenadine.

\section{Conclusions}

The current metabolomic study has successfully identified and characterized seven potential biomarkers in the plasma of untreated GD patients using rapid SPE purification followed by a UPLC-TOF/MS analysis. As previously mentioned, some were already identified but not characterized by fragmentation studies. In summary, out of the 91,197 markers observed in plasma samples of GD patients and age- and gender-matched controls, lyso-Gb $\mathrm{b}_{1}$ and four related analogs $(-28 \mathrm{Da},-2 \mathrm{Da}$, +14 Da, and +18 Da), SPC, and N-palmitoyl-O-phosphocholineserine were highlighted as relevant to the disease by multivariate statistical analyses, such as OPLS-DA and S-plot charts. The lyso-Gb analog +18 Da has never been described in the literature, while lyso-Gb $\mathrm{b}_{1}$ analogs $-28 \mathrm{Da},-2 \mathrm{Da}$, and +14 Da have never been characterized using chromatographic separation or mass spectrometry fragmentation tests.

Although lyso-Gb b $_{1}$ SPC, and N-palmitoyl-O-phosphocholineserine have previously been observed in GD plasma samples, the metabolomic study has demonstrated the importance of evaluating a group of potential biomarkers to achieve a high level of sensitivity for a potential GD diagnostic test. Indeed, patient ID 14 did not have significantly elevated plasma levels of lyso-Gb $b_{1}$ or its related analogs. However, he did have increased levels of N-palmitoyl-O-phosphocholineserine. Therefore, a potential diagnostic test based solely on lyso- $\mathrm{Gb}_{1}$ would have provided a false negative result for this latter patient. This emphasizes the importance of having a diagnostic test based on a profile of biomarkers to be analyzed rather than only one biomarker. Considering that two biomarkers are not specific for GD (SPC and N-palmitoyl-O-phosphocholineserine) since they can be detected in other LSDs, we strongly recommend that these seven biomarkers be part of a biomarker profile for GD diagnosis, thus improving the overall specificity. In fact, having biomarkers that are not specific for GD highlights the importance of performing the enzyme analysis or molecular testing to confirm the diagnosis. However, the suggestion to use a profile of biomarkers might help for the early detection of GD patients. Potential test results, where N-palmitoyl-O-phosphocholineserine and SPC are increased, while lyso-G $b_{1}$ and its related analogs are not detected, might lead to a false differential diagnosis. While biomarker quantitation analysis is often the first test performed for potential GD patients, a thorough enzyme activity testing and molecular genetics analyses are necessary to confirm the diagnosis. Future perspectives will involve the development of a novel validated method for the quantitation of these biomarkers using a tandem mass spectrometry approach to thoroughly investigate potential correlations between biomarker concentrations and age, as well as clinical manifestations in treated and untreated GD patients for additional insights into the pathophysiology of the disease.

\section{Study Limitations}

Study limitations include the small number of untreated GD patients, as well as a low number of different mutations. 
Author Contributions: I.M. was involved in the sample preparation, analysis, and data processing. I.M. was also involved in the original draft preparation, as well as the review and editing process. M.B. was involved in the supervision of the study and the interpretation of results, in the drafting and revision of the manuscript. A.A., F.E.M. and G.-É.R. provided the samples used for this study, as well as pertinent patient clinical information. They were also involved in the revision of the manuscript. C.A.-B. was involved in the design and supervision of the study and in the interpretation of results. C.A.-B. also facilitated the recruitment of specimens from healthy controls and GD patients and secured the resources and funding required for this study. C.A.-B. was also involved in the drafting and revision of the manuscript. All authors have read and agreed to the published version of the manuscript.

Funding: This research was funded by Shire/Takeda Pharmaceutical.

Acknowledgments: We would like to acknowledge Shire/Takeda Pharmaceutical for funding this research project. We are grateful to the Faculty of Medicine and Health Sciences at the Universite de Sherbrooke for a doctoral student grant for Iskren Menkovic. We would also like to thank Waters Corporation for their technical expertise and continuous support.

Conflicts of Interest: F.E.M. was paid for an advisory board by Sanofi-Genzyme. A.A. and G.-É.R. declare that they have no conflict of interest. I.M. received a student stipend from Shire/Takeda Pharmaceutical for this project. M.B. received a salary. C.A.-B. received research grants and honoraria from Shire/Takeda Pharmaceutical.

\section{Appendix A}
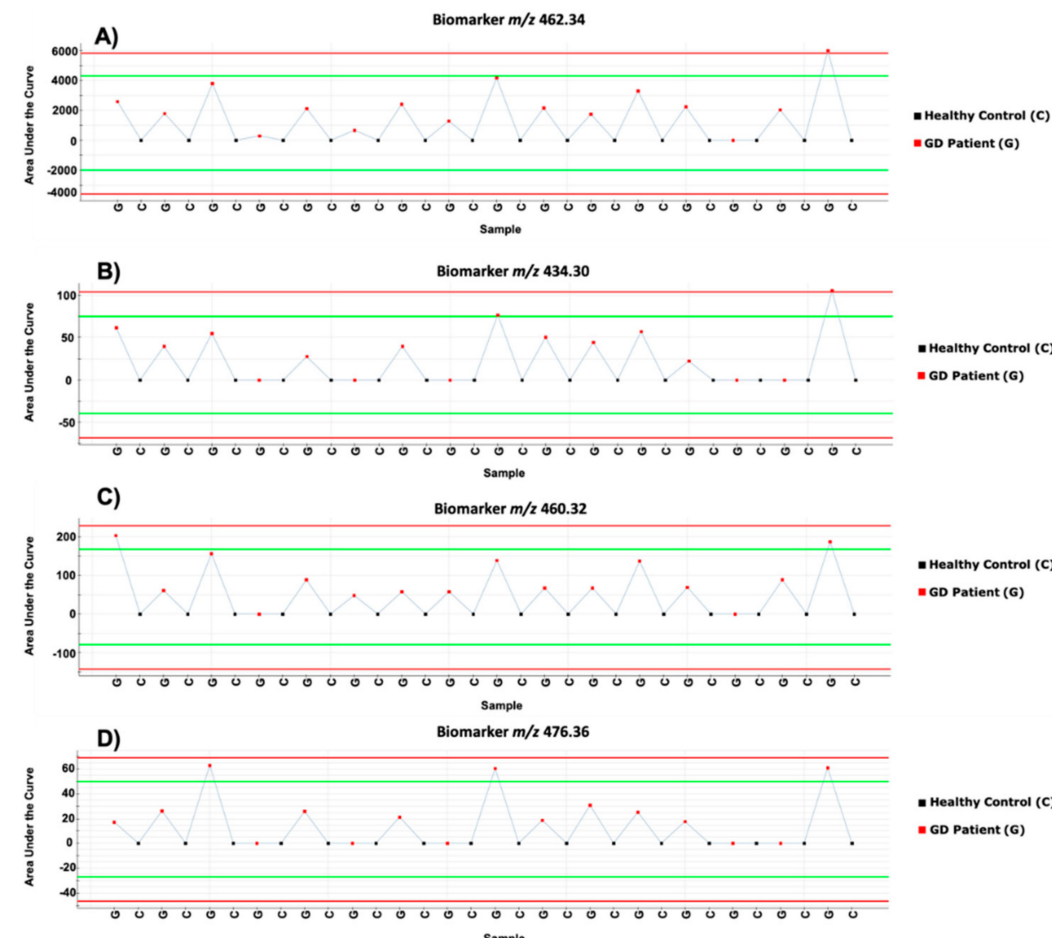

Figure A1. Cont. 


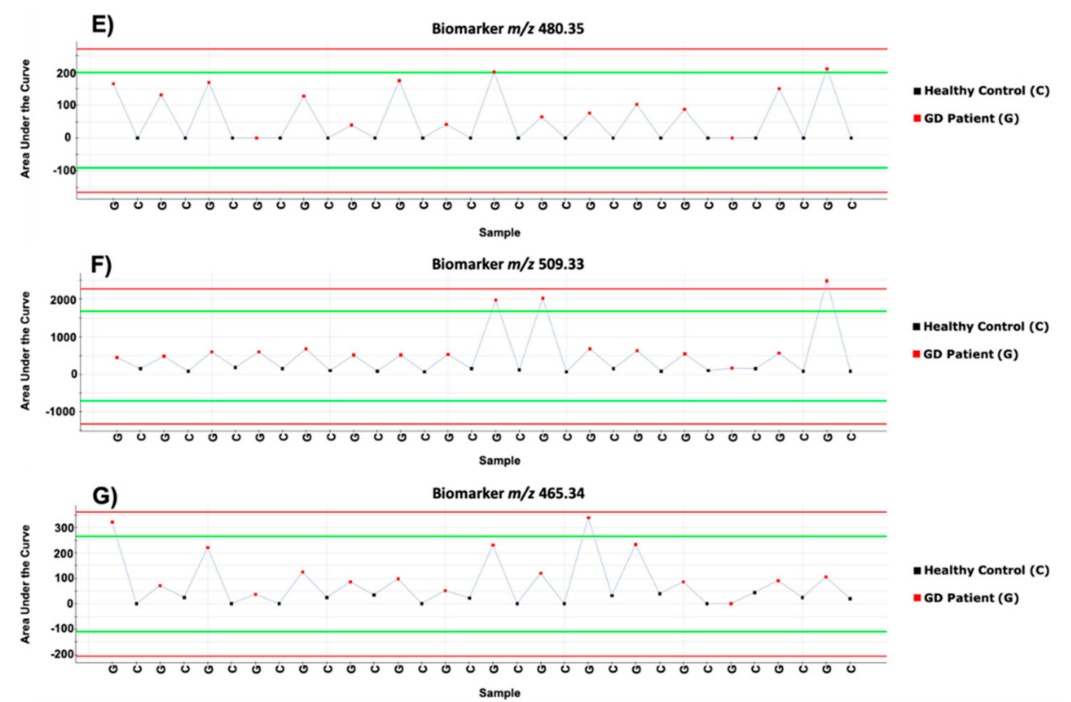

Figure A1. Trend plot charts for lyso-Gb $\mathrm{G}_{1}$ (A), lyso-Gb $\mathrm{b}_{1}$ analogs $-28 \mathrm{Da}$ (B), $-2 \mathrm{Da}(\mathbf{C})$, +14 Da (D), +18 Da (E), N-palmitoyl-O-phosphocholineserine (F), and sphingosylphosphorylcholine (G). $\mathrm{G}=$ Gaucher patient, $\mathrm{C}=$ healthy control.
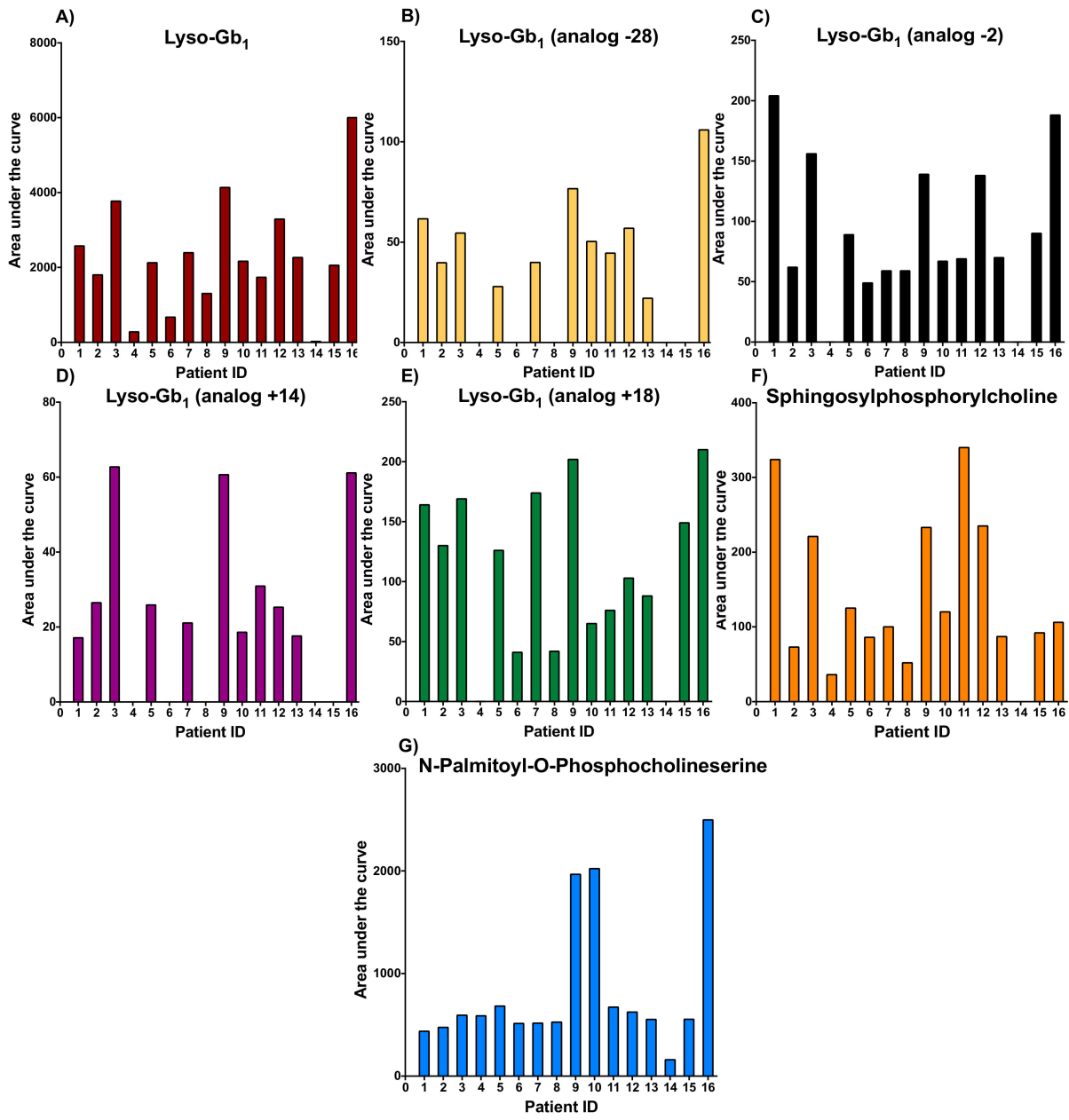

Figure A2. Areas under the curve are shown for lyso-Gb 1 (A), lyso-Gb 1 analog $+18 \mathrm{Da}(\mathbf{B}), \mathrm{lyso}_{1}-\mathrm{Gb}_{1}$ analog $-2 \mathrm{Da}(\mathbf{C})$, lyso-Gb 1 analog $-28 \mathrm{Da}(\mathbf{D})$, lyso-Gb 1 analog +14 Da (E), sphingosylphosphorylcholine $(\mathbf{F})$, and N-palmitoyl-O-phosphocholineserine $(\mathrm{G})$. These biomarkers were not detected in control samples. 


\section{References}

1. Smith, L.; Mullin, S.; Schapira, A. Insights into the structural biology of Gaucher disease. Exp. Neurol. 2017, 298 Pt B, 180-190. [CrossRef]

2. Aerts, J.M.; Kuo, C.L.; Lelieveld, L.T.; Boer, D.E.; Lienden, M.J.V.D.; Overkleeft, H.S.; Artola, M. Glycosphingolipids and lysosomal storage disorders as illustrated by Gaucher disease. Curr. Opin. Chem. Biol. 2019, 53, 204-215. [CrossRef] [PubMed]

3. Andrade-Campos, M.; Alfonso, P.; Irun, P.; Armstrong, J.; Calvo, C.; Dalmau, J.; Domingo, M.R.; Barbera, J.L.; Cano, H.; Fernandez-Galán, M.A.; et al. Diagnosis features of pediatric Gaucher disease patients in the era of enzymatic therapy, a national-base study from the Spanish Registry of Gaucher disease. Orphanet. J. Rare Dis. 2017, 12. [CrossRef] [PubMed]

4. Zeid, N.; Stauffer, C.; Yang, A.; Naik, H.; Fierro, L.; Ganesh, J.; Balwani, M. The N370S/R496H genotype in type 1 Gaucher disease-Natural history and implications for pre-symptomatic diagnosis and counseling. Mol. Genet. Metab. Rep. 2020, 22, 100567. [CrossRef] [PubMed]

5. Stirnemann, J.; Belmatoug, N.; Camou, F.; Serratrice, C.; Froissart, R.; Caillaud, C.; Levade, T.; Astudillo, L.; Serratrice, J.; Brassier, A.; et al. Review of Gaucher disease pathophysiology, clinical presentation and treatments. Int. J. Mol. Sci. 2017, 18, 441. [CrossRef] [PubMed]

6. Fraga, C.; Medeiros, S.; Serpa, S.; Silva, D. Case Series Synopsis: Gaucher disease type 1 patients treated with eliglustat over 6 years. Int. J. Rare Dis. Disord. 2020, 3. [CrossRef]

7. Mistry, P.K.; Lopez, G.; Schiffmann, R.; Barton, N.W.; Weinreb, N.J.; Sidransky, E. Gaucher disease: Progress and ongoing challenges. Mol. Genet. Metab. 2017, 120, 8-21. [CrossRef]

8. Dandana, A.; Khelifa, S.B.; Chahed, H.; Miled, A.; Ferchichi, S. Gaucher disease: Clinical, biological and therapeutic aspects. Pathobiology 2015, 83, 13-23. [CrossRef]

9. Lal, T.R.; Sidransky, E. The spectrum of neurological manifestations associated with Gaucher disease. Diseases 2017, 5, 10. [CrossRef]

10. Schiffmann, R.; Sevigny, J.; Rolfs, A.; Davies, E.H.; Goker-Alpan, O.; Abdelwahab, M.; Vellodi, A.; Mengel, E.; Lukina, E.; Yoo, H.W.; et al. The definition of neuronopathic Gaucher disease. J. Inherit. Metab. Dis. 2020, 1-4. [CrossRef]

11. Van Rossum, A.; Holsopple, M. Enzyme replacement or substrate reduction? A Review of Gaucher disease treatment options. Hosp. Pharm. 2016, 51, 553-563. [CrossRef]

12. Gary, S.E.; Ryan, E.; Steward, A.M.; Sidransky, E. Recent advances in the diagnosis and management of Gaucher disease. Expert Rev. Endocrinol. Metab. 2018, 13, 107-118. [CrossRef]

13. Giraldo, P.; Frutos, L.L.D.; Cebolla, J.J. Biomarker combination is necessary for the assessment of Gaucher disease? Ann. Transl. Med. 2018, 6. [CrossRef] [PubMed]

14. Raskovalova, T.; Deegan, P.B.; Yang, R.; Pavlova, E.; Stirnemann, J.; Labarère, J.; Zimran, A.; Mistry, P.; Berger, M. Plasma chitotriosidase activity versus CCL18 level for assessing type I Gaucher disease severity: Protocol for a systematic review with meta-analysis of individual participant data. Syst. Rev. 2017, 6. [CrossRef] [PubMed]

15. Bernstein, K.E.; Khan, Z.; Giani, J.F.; Cao, D.Y.; Bernstein, E.A.; Shen, X.Z. Angiotensin-converting enzyme in innate and adaptive immunity. Nat. Rev. Nephrol. 2018, 14, 325-336. [CrossRef] [PubMed]

16. Van Dussen, L.; Lips, P.; Everts, V.E.; Bravenboer, N.; Jansen, I.D.; Groener, J.E.; Maas, M.; Blokland, J.A.; Aerts, J.M.; Hollak, C.E. Markers of bone turnover in Gaucher disease: Modeling the evolution of bone disease. J. Clin. Endocrinol. Metab. 2011, 96, 2194-2205. [CrossRef]

17. Murugesan, V.; Chuang, W.L.; Liu, J.; Lischuk, A.; Kacena, K.; Lin, H.; Pastores, G.M.; Yang, R.; Keutzer, J.; Zhang, K.; et al. Glucosylsphingosine is a key biomarker of Gaucher disease. Am. J. Hematol. 2016, 91, 1082-1089. [CrossRef]

18. Ferraz, M.J.; Marques, A.R.; Appelman, M.D.; Verhoek, M.; Strijland, A.; Mirzaian, M.; Scheij, S.; Ouairy, C.M.; Lahav, D.; Wisse, P.; et al. Lysosomal glycosphingolipid catabolism by acid ceramidase: Formation of glycosphingoid bases during deficiency of glycosidases. FEBS Lett. 2016, 590, 716-725. [CrossRef]

19. Rolfs, A.; Giese, A.K.; Grittner, U.; Mascher, D.; Elstein, D.; Zimran, A.; Böttcher, T.; Lukas, J.; Hübner, R.; Gölnitz, U.; et al. Glucosylsphingosine is a highly sensitive and specific biomarker for primary diagnostic and follow-up monitoring in Gaucher disease in a non-Jewish, Caucasian cohort of Gaucher disease patients. PLoS ONE 2013, 8, e79732. [CrossRef] [PubMed] 
20. Dekker, N.; van Dussen, L.; Hollak, C.E.; Overkleeft, H.; Scheij, S.; Ghauharali, K.; van Breemen, M.J.; Ferraz, M.J.; Groener, J.E.; Maas, M.; et al. Elevated plasma glucosylsphingosine in Gaucher disease: Relation to phenotype, storage cell markers, and therapeutic response. Blood 2011, 118, e118-e127. [CrossRef]

21. Arkadir, D.; Dinur, T.; Revel-Vilk, S.; Becker Cohen, M.; Cozma, C.; Hovakimyan, M.; Eichler, S.; Rolfs, A.; Zimran, A. Glucosylsphingosine is a reliable response biomarker in Gaucher disease. Am. J. Hematol. 2018, 93, E140-E142. [CrossRef] [PubMed]

22. Mucci, J.M.; Rozenfeld, P. Pathogenesis of bone alterations in Gaucher disease: The role of immune System. J. Immunol. Res. 2015, 2015, 192761. [CrossRef] [PubMed]

23. Irún, P.; Cebolla, J.J.; López de Frutos, L.; De Castro-Orós, I.; Roca-Espiau, M.; Giraldo, P. LC-MS/MS analysis of plasma glucosylsphingosine as a biomarker for diagnosis and follow-up monitoring in Gaucher disease in the Spanish population. Clin. Chem. Lab. Med. 2020, 58, 798-809. [CrossRef] [PubMed]

24. Cozma, C.; Cullufi, P.; Kramp, G.; Hovakimyan, M.; Velmishi, V.; Gjikopulli, A.; Tomori, S.; Fischer, S.; Oppermann, S.; Grittner, U.; et al. Treatment efficiency in Gaucher patients can reliably be monitored by quantification of Lyso-Gb1 concentrations in dried blood spots. Int. J. Mol. Sci. 2020, 21, 4577. [CrossRef]

25. Dinur, T.; Zimran, A.; Becker-Cohen, M.; Arkadir, D.; Cozma, C.; Hovakimyan, M.; Oppermann, S.; Demuth, L.; Rolfs, A.; Revel-Vilk, S. Long term follow-up of 103 untreated adult patients with type 1 Gaucher disease. J. Clin. Med. 2019, 8, 1662. [CrossRef]

26. Lavoie, P.; Boutin, M.; Auray-Blais, C. Multiplex analysis of novel urinary lyso-Gb $b_{3}$-related biomarkers for Fabry disease by tandem mass spectrometry. Anal Chem. 2013, 85, 1743-1752. [CrossRef]

27. Boutin, M.; Auray-Blais, C. Multiplex tandem mass spectrometry analysis of novel plasma lyso-Gb ${ }_{3}$-related analogues in Fabry disease. Anal Chem. 2014, 86, 3476-3483. [CrossRef] [PubMed]

28. Boutin, M.; Auray-Blais, C. Metabolomic Discovery of novel urinary galabiosylceramide analogs as Fabry disease biomarkers. J. Am. Soc. Mass. Spectrom. 2015, 26, 499-510. [CrossRef] [PubMed]

29. Auray-Blais, C.; Boutin, M.; Gagnon, R.; Dupont, F.O.; Lavoie, P.; Clarke, J.T. Urinary globotriaosylsphingosine-related biomarkers for Fabry disease targeted by metabolomics. Anal Chem. 2012, 84, 2745-2753. [CrossRef] [PubMed]

30. Mirzaian, M.; Wisse, P.; Ferraz, M.J.; Gold, H.; Donker-Koopman, W.E.; Verhoek, M.; Overkleeft, H.S.; Boot, R.G.; Kramer, G.; Dekker, N.; et al. Mass spectrometric quantification of glucosylsphingosine in plasma and urine of type 1 Gaucher patients using an isotope standard. Blood Cells Mol. Dis. 2015, 54, 307-314. [CrossRef] [PubMed]

31. O'Sullivan, C.; Dev, K.K. Galactosylsphingosine (psychosine)-induced demyelination is attenuated by sphingosine 1-phosphate signalling. J. Cell Sci. 2015, 128, 3878-3887. [CrossRef] [PubMed]

32. Herbst, Z.; Turgeon, C.T.; Biski, C.; Khaledi, H.; Shoemaker, N.B.; Dearmond, P.D.; Smith, S.; Orsini, J.; Matern, D.; Gelb, M.H. Achieving congruence among reference laboratories for absolute abundance measurement of analytes for rare diseases: Psychosine for diagnosis and prognosis of Krabbe disease. Int. J. Neonatal. Screen 2020, 6, 29. [CrossRef] [PubMed]

33. Boutin, M.; Sun, Y.; Shacka, J.J.; Auray-Blais, C. Tandem mass spectrometry multiplex analysis of glucosylceramide and galactosylceramide isoforms in brain tissues at different stages of Parkinson disease. Anal Chem. 2016, 88, 1856-1863. [CrossRef] [PubMed]

34. Sidhu, R.; Mondjinou, Y.; Qian, M.; Song, H.; Kumar, A.B.; Hong, X.; Hsu, F.F.; Dietzen, D.J.; Yanjanin, N.M.; Porter, F.D.; et al. N-acyl-O-phosphocholineserines: Structures of a novel class of lipids that are biomarkers for Niemann-Pick C1 disease. J. Lipid Res. 2019, 60, 1410-1424. [CrossRef]

35. Sidhu, R.; Kell, P.; Dietzen, D.J.; Farhat, N.Y.; Do, A.; Porter, F.D.; Berry-Kravis, E.; Vite, C.H.; Reunert, J.; Marquardt, T.; et al. Application of N-palmitoyl-O-phosphocholineserine for diagnosis and assessment of response to treatment in Niemann-Pick type C disease. Mol. Genet. Metab. 2020, 129, 292-302. [CrossRef]

36. Thomas, G.D.; Snetkov, V.A.; Patel, R.; Leach, R.M.; Aaronson, P.I.; Ward, J.P. Sphingosylphosphorylcholine-induced vasoconstriction of pulmonary artery: Activation of non-store-operated $\mathrm{Ca}^{2+}$ entry. Cardiovasc. Res. 2005, 68, 56-64. [CrossRef]

37. Ługowska, A.; Hetmańczyk-Sawicka, K.; Iwanicka-Nowicka, R.; Fogtman, A.; Cieśla, J.; Purzycka-Olewiecka, J.K.; Sitarska, D.; Płoski, R.; Filocamo, M.; Lualdi, S.; et al. Gene expression profile in patients with Gaucher disease indicates activation of inflammatory processes. Sci. Rep. 2019, 9, 6060. [CrossRef] 
38. Deodato, F.; Boenzi, S.; Taurisano, R.; Semeraro, M.; Sacchetti, E.; Carrozzo, R.; Dionisi-Vici, C. The impact of biomarkers analysis in the diagnosis of Niemann-Pick $C$ disease and acid sphingomyelinase deficiency. Clin. Chim. Acta 2018, 486, 387-394. [CrossRef]

39. Zhang, K.; Chuang, W.-L.; Pacheco, J.; Cooper, S.; Mcgovern, M.; Cox, G.; Keutzer, J. Lyso-sphingomyelin is elevated in dried blood spots of Niemann-Pick disease type B patients. Mol. Genet. Metab. 2014, 111, $209-211$. [CrossRef]

40. Saville, J.T.; McDermott, B.K.; Chin, S.J.; Fletcher, J.M.; Fuller, M. Expanding the clinical utility of glucosylsphingosine for Gaucher disease. J. Inherit. Metab. Dis. 2020, 43, 558-563. [CrossRef]

41. Hurvitz, L.; Dinur, T.; Becker-Cohen, M.; Cozma, C.; Hovakimyan, M.; Oppermann, S.; Demuth, L.; Rolfs, A.; Abramov, A.; Zimran, A.; et al. Glucosylsphingosine (lyso-Gb $\left.\mathrm{G}_{1}\right)$ as a Biomarker for Monitoring Treated and Untreated Children with Gaucher Disease. Int. J. Mol. Sci. 2019, 20, 3033. [CrossRef] [PubMed]

42. Giese, A.K.; Mascher, H.; Grittner, U.; Eichler, S.; Kramp, G.; Lukas, J.; te Vruchte, D.; Al Eisa, N.; Cortina-Borja, M.; Porter, F.D.; et al. A novel, highly sensitive and specific biomarker for Niemann-Pick type C1 disease. Orphanet. J. Rare Dis. 2015, 10, 78. [CrossRef]

43. Auray-Blais, C.; Lavoie, P.; Boutin, M.; Ntwari, A.; Hsu, T.; Huang, C.; Niu, D. Biomarkers associated with clinical manifestations in Fabry disease patients with a late-onset cardiac variant mutation. Clin. Chim. Acta 2017, 466, 185-193. [CrossRef] [PubMed]

44. Van den Berg, R.A.; Hoefsloot, H.C.; Westerhuis, J.A.; Smilde, A.K.; van der Werf, M.J. Centering, scaling, and transformations: Improving the biological information content of metabolomics data. BMC Genom. 2006, 7, 142. [CrossRef] [PubMed]

Publisher's Note: MDPI stays neutral with regard to jurisdictional claims in published maps and institutional affiliations. 